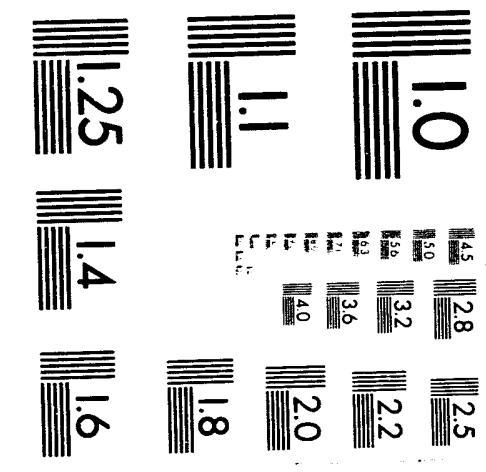



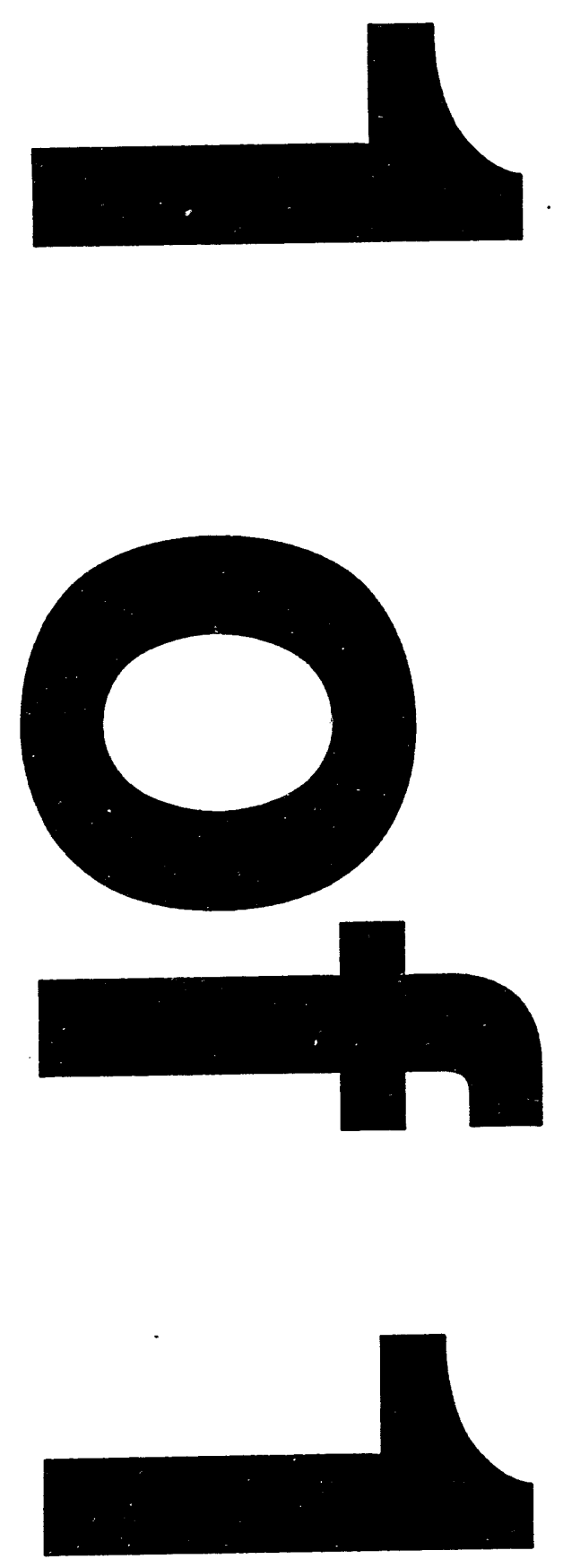


\title{
DYNAMIC MODELING AND RESPONSE OF SOIL-WALL SYSTEMS
}

\author{
A.S. Veletsos and A.H. Younan \\ Rice University, Houston, Texas
}

Under contract to

Engineering Research \& Applications Division

Department of Advanced Technology

Brookhaven National Laboratory

P.O. Box 5000

Upton, N.Y. 11973-5000

October 1993

Prepared for

OFFICE OF ENVIRONMENTAL RESTORATION AND WASTE MANAGEMENT DEPARTMENT OF ENERGY, WASHINGTON, D.C. 


\section{DISCLAIMER}

This report was prepared as an account of work sponsored by an agency of the United States Government. Neither the United States Government nor any agency thereof, nor any of their employees, nor any of their contractors, subcontractors, or their employees, makes any warranty, express or implied, or assumes any legal liability or responsibility for the accuracy, completeness, or usefulness of any information, apparatus, product, or process disclosed, or represents that its use would not infringe privately owned rights. Reference herein to any specific commercial product, process, or service by trade name, trademark, manufacturer, or otherwise, does not necessarily constitute or imply its endorsement, recommendation, or favoring by the United States Government or any agency, contractor or subcontractor thereof. The views and opinions of authors expressed herein do not necessarily state or reflect those of the United States Government or any agency, contractor or subcontractor thereof.

\section{Printed in the United States of America}

Available from

National Technical Information Service

U.S. Department of Commerce

5285 Port Royal Road

Springfield, VA 22161

NTIS price codes:

Printed Copy: A04; Microfiche Copy: A01 


\begin{abstract}
Following a brief review of the errors that may result from the use of a popular model for evaluating the dynamic soil forces induced in a base-excited rigid wall retaining an elastic stratum, the sources of the errors are identified and a modification is proposed which defines correctly the action of the system. In the proposed modification, the stratum is modeled by a series of elastically supported, semi-infinite horizontal bars with distributed mass instead of massless springs. The concepts involved are introduced by reference to a system composed of a fixed-based wall and a homogeneous elastic stratum, and are then applied to the analysis of more complex soil-wall systems. Both harmonic and transient excitations are considered, and comprehensive numerical solutions are presented which elucidate the actions involved and the effects and relative importance of the relevant parameters.
\end{abstract}




\section{TABLE OF CONTENTS}

Section

Page

ABSTRACT

iii

TABLE OF CONTENTS $v$

LIST OF FIGURES vii

EXECUTIVE SUMMARY ix

ACKNOWLEDGMENT

1 INTRODUCTION $1-1$

2 SYSTEM CONSIDERED 2-1

3 SCOTT'S MODEL AND ITS ACCURACY 3-1

3.1 A Modification of Scott's Model 3-2

4 PROPOSED MODEL 4-1

5 FUNDAMENTAL SOLUTIONS FOR ELASTICALLY SUPPORTED BARS 5-1

5.1 Bar Impedance $\quad 5-1$

6 HOMOGENEOUS LAYER WITH IRROTATIONAL WALL 6-1

6.1 Harmonic Response 6-1

6.2 Transient Response $\quad 6-2$

6.3 Numerical Results 6-3

7 HOMOGENEOUS LAYER WITH ELASTICALLY CONSTRAINED WALL 7-1

$\begin{array}{ll}7.1 \text { Numerical Solutions } & \text { 7-2 }\end{array}$

8 INHOMOGENEOUS LAYER WITH ELASTICALLY CONSTRAINED WALL 8-1

9 CONCLUSIONS 9-1

10 REFERENCES 10-1

11 NOTATION 11-1 


\section{LIST OF FIGURES}

Figure

Page

2.1 System considered. $2-3$

3.1 Scott's model. $3-4$

3.2 Frequency response curves for amplitude of base shear in fixed-based wall with $v=1 / 3$ and $\delta=0.1$.

4.1 Exact modeling of soil stratum.

5.1 Stiffness and damping coefficient for semi-infinite elastically constrained bar.

7.1 Effect of rocking flexibility of wall on distribution of wall pressures for statically excited systems; uniform stratum with $v=1 / 3$.

7.2 Effect of rocking flexibility of wall on base shear and moment in wall of statically excited systems; uniform stratum with $v=1 / 3$.

7.3 Effect of rocking flexibility of wall on base shear of harmonically excited systems; uniform stratum with $v=1 / 3$ and $\delta=0.1$.

7.4 Effect of rocking flexibility of wall on maximum base shear in wall of systems subjected to El Centro record; uniform stratum with $v=1 / 3$ and $\delta=0.1$.

8.1 Modes of vibration of a cantilever shear-beam with a parabolic variation of shear modulus.

8.2 Distribution of wall pressures for systems with a rotationally constrained wall and a medium with parabolic variation in shear modulus; $v=1 / 3$.

8.3 Base shear and base moment for systems with a rotationally constrained wall and a medium with parabolic variation in shear modulus; $v=1 / 3$.

8.4 Frequency response curves for amplitude of base shear in wall of systems with uniform, and parabolic variations in shear modulus; $v=1 / 3$ and $\delta=0.1$. 


\section{EXECUTIVE SUMMARY}

The study reported herein is the third in a series of investigations motivated by the need to gain improved understanding of the responses to earthquakes of deeply embedded and underground tanks storing radioactive wastes, and to develop rational but simple methods of analysis and design for such systems. The first two studies were reported in Brookhaven National Laboratory reports 52357 and 52372.

Probably the simplest available approximate procedure for evaluating the dynamic soil pressures induced by ground shaking on retaining walls and on embedded structures is the one proposed in 1973 by Scott. In this approach, the far-field response of the medium is evaluated considering it to respond as a base-excited, vertical cantilever shear-beam, and the dynamic wall pressure at a given height is taken proportional to the difference in motions of the shear-beam and the wall at that height. This is tantamount to representing the restraining action of the medium by a series of massless, linear horizontal springs.

Following a brief review of the errors that may result from the use of this approach, the sourcas of the errors are identified and a modification is proposed which defines correctly the action of the system. In the proposed modification, the stratum is modeled by a series of elastically supported, semi-infinite horizontal bars with distributed mass instead of massless springs. The concepts involved are introduced by reference to a system composed of a fixed-based wall and a homogeneous elastic stratum, and are then applied to the analysis of systems with a wall that is elastically constrained against rotation at its base and a medium for which the shear modulus is either uniform or increases parabolically with depth. Both harmonic and transient excitations are considered, and comprehensive numerical solutions are presented which elucidate the actions involved and the effects and relative importance of the relevant parameters.

The principal conclusions of the study may be summarized as follows:

1. The errors in Scott's model stem from its failure to provide for the radiational damping of the medium and its capacity to transfer forces vertically by horizontal shearing action.

2. The deficiencies of this model may be eliminated by modeling the restraining action of the medium by a series of elastically supported, semi-infinite horizontal bars with distributed mass rather than by massless springs. The impedance of each of these bars depends on the ratio of the exciting fre- 
quency to the natural frequency of the medium for the mode of vibration being considered, and the material damping factor of the medium. The response of the bars may be evaluated either in the frequency domain by use of Fourier transform techniques or directly in the time-domain by use of a convolution integral analogous to Duhamel's integral for single-degree-of-freedom systems.

3. The rotational flexibility of the wall decreases the dynamic wall pressures and the associated shears and bending moments and affects dramatically their distributions.

4. The greater the wall flexibility, the greater is the number of modes for the medium required to accurately represent the distribution of 11 pressures. However, reasonable approximations to the base forces in the wall may still be obtained considering the contribution of only the first two modes of vibration for shear and of only the first mode for moment.

5. The magnitude and distribution of the wall pressures are also sensitive to the depthwise variation of the shear modulus for the medium.

6. The comprehensive numerical solutions presented and their analysis provide not only valuable insights into the responses of the systems examined and into the effects and relative importance of the numerous parameters involved, but also a conceptual framework for the interpretation of the responses of still more complex soil-wall systems. 


\section{ACKNOWLEDGEMENT}

This study was carried out at Rice University under Grant 568821 from the Brookhaven National Laboratory, Upton, New York. This support is acknowledged gratefully. Appreciation also is expressed to Drs. M. Reich and K. Bandyopadhyay for helpful comments and their understanding project management. 


\section{SECTION 1}

\section{INTRODUCTION}

Probably the simplest available approximate procedure for evaluating the dynamic soil pressures induced by ground shaking on retaining walls and on embedded structures is the one proposed by Scott (1973). In this approach, the far-field response of the medium is evaluated considering it to respond as a base-excited, vertical cantilever shear-beam, and the dynamic wall pressure at a given height is taken proportional to the relative motions of the shear-beam and the wall at that height. This is tantamount to representing the restraining action of the medium by a series of massless, linear horizontal springs.

Scott's model has been used extensively (e.g., Karkanias 1983, Dennehy 1984, Jain and Scott 1989 , Alampalli and Elgamal 1991, Soydemir 1991) and variations of it have been employed in analyses of embedded foundations (Beredugo and Novak 1972, Novak and Beredugo 1972), piles (Novak 1974, Flores-Berrones and Whitman 1982) and underground cylindrical structures (Miller et al 1991). Proposed originally for an elastic soil stratum retained along its vertical boundaries by a pair of rigid walls, the procedure is also applicable to the important limiting case of a semi-infinite stratum retained at one end. The procedure has been applied to rigid and flexible walls as well as to systems for which the soil modulus increases with depth (Scott 1973, Jain and Scott 1989).

In a recent study of the effects of ground shaking on rigid walls retaining a semi-infinite, viscoelastic stratum (Veletsos and Younan 1992), it has been shown that, depending on the characteristics of the ground motion, Scott's model may lead to significant errors. The objectives of the present study are to: (1) highlight the nature of the inaccuracies that may result; (2) identify their source or sources; (3) present a modification which correctly describes the action of the system; and (4) use the modified model for the analysis of different soil-wall systems.

The system investigated is a semi-infinite, viscoelastic stratum of constant thickness that is supported on a non-deformable rigid base and is retained along one of its vertical boundaries by a rigid wall that is either fixed or elastically constrained against rotation at the base. Both the wall base and the base of the stratum are presumed to be excited by a space-invariant, uniform horizontal motion. The concepts involved are first developed by reference to a uniform stratum and a wall that is fixed against rotation at its base, and are then applied to the analysis of systems composed of an elastically constrained wall 
and a vertically inhomogeneous medium. The effects of both harmonic and transient excitations are examined, with special attention paid to the important limiting case of systems excited by very-lowfrequency, essentially static base motions. Comprehensive numerical solutions are presented which elucidate the actions of the systems considered and the effects and relative importance of the numerous parameters involved. 


\section{SECTION 2}

\section{SYSTEM CONSIDERED}

The system examined is shown in part (a) of Fig. 2-1. It is a semi-infinite, uniform layer of linear viscoelastic material that is free at its upper surface, is bonded to a non-deformable, rigid base, and is retained along one of its vertical boundaries by a rigid wall. The heights of the wall and stratum are considered to be the same and they are denoted by $\mathrm{H}$. The wall may be either fixed or elastically constrained against rotation at its base, the stiffness of the rotational constraint being denoted by $R_{\boldsymbol{\theta}}$. The bases of both the stratum and the wall are presumed to experience a space-invariant horizontal motion, the acceleration of which at any time $t$ is $\ddot{x}_{B}(t)$. Material damping for the medium is considered to be of the constant hysteretic type.

The properties of the layer are defined by its mass density, $\rho$, shear modulus of elasticity, G, Poisson's ratio, $v$, and the material damping factor, $\delta$, which is considered to be frequency-independent and the same for both shearing and axial deformations. The latter factor is the same as the $\tan \delta$ factor used by the senior author and his associates in studies of foundation dynamics and soil-structure interaction (e.g., Veletsos and Verbic 1973, Veletsos and Nair 1975), and twice as large as the percentage of critical damping. $\beta$, used by other authors in related studies (e.g., Wood 1973, Scott 1973, Arias et al 1981).

The displacements relative to the moving boundary and the resulting wall pressures and forces for the base-excited system can be shown to be identical to those of the force-excited system indicated in part (b) of Fig. 2-1. Both the wall and the base of the latter system are stationary, and the stratum is acted upon by uniform lateral body forces of intensity $-p \ddot{x}_{g}(t)$. The action of the force-excited system may in some instances be easier to visualize than that of the base-excited system. 


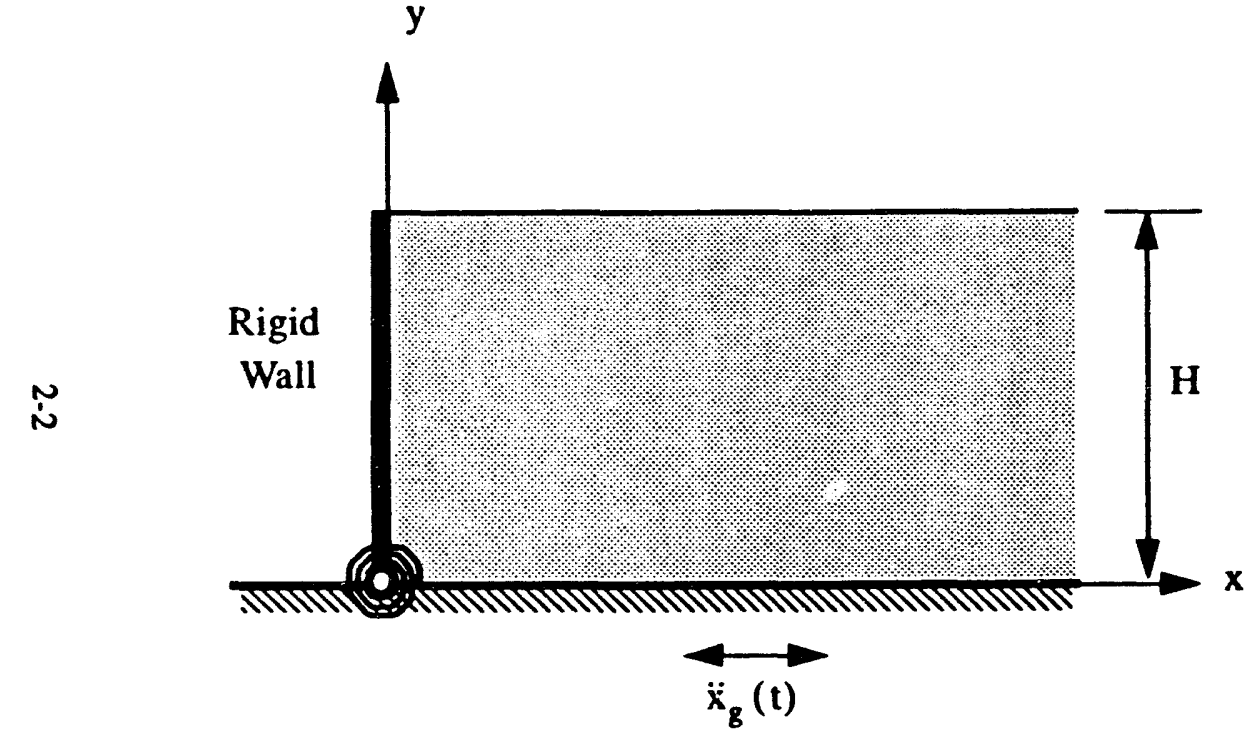

(a) Ground-Excited

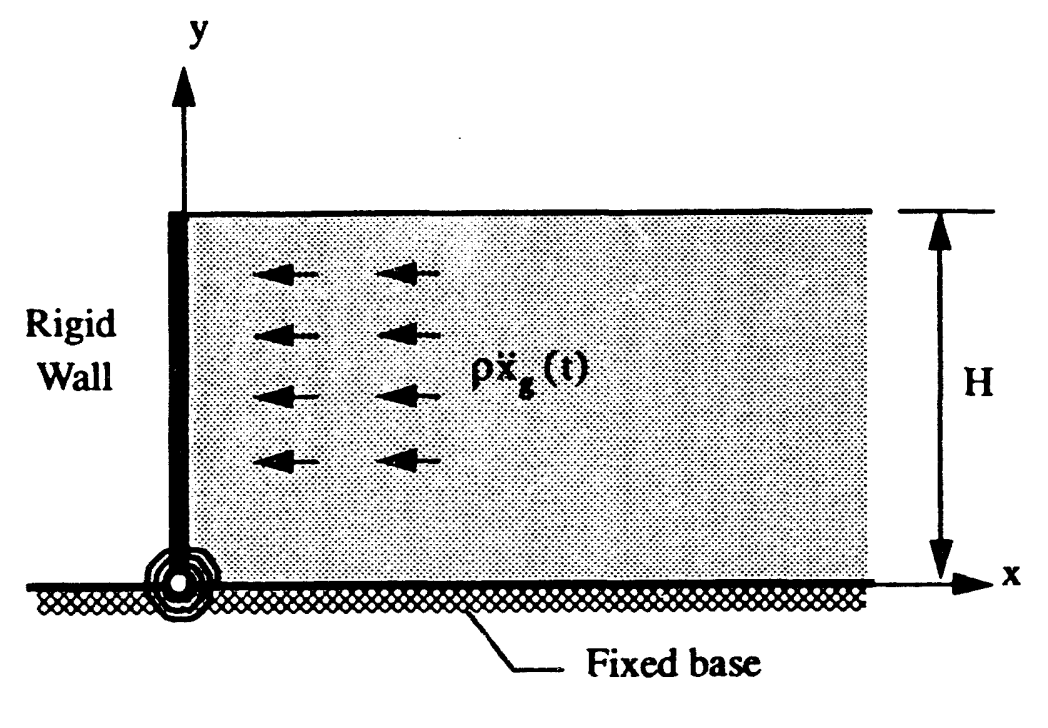

(b) Force-Excited

Fig. 2-1 System considered 


\section{SECTION 3}

\section{SCOTT'S MODEL AND ITS ACCURACY}

Scott's model for the system is shown in Fig. 3-1. It consists of a vertical cantilever shear-beam that simulates the far-field action of the stratum, and a set of massless, linear horizontal springs connecting the shear-beam to the wall. The height and material properties of the beam are taken equal to those of the stratum, and the stiffness of the springs per unit of length and height of the wall, $\mathrm{K}_{\mathrm{s}}$, is taken as

$$
\mathrm{K}_{\mathrm{s}}=\frac{0.8(1-\mathrm{v})}{1-2 \mathrm{~V}} \frac{\mathrm{G}}{\mathrm{H}}
$$

This stiffness is the same as the extensional stiffness of a bar of unit cross sectional area and length $2.5 \mathrm{H}$ that is fully constrained along its sides. The bases of both the shear-beam and the wall are presumed to be excited by the same ground motion. The wall pressure at a given height is thus given by the product of $\mathrm{K}$, and the relative motions of the shear-beam and the wall at that height. Note that the spring stiffness is independent of the characteristics of the ground motion and that the only damping for the model is that involved in the shear-beam itself. Note further that as $v \rightarrow 0.5, \mathrm{~K}_{\mathrm{s}}$ and hence the wall pressures and associated forces for the wall become infinite, a result that is clearly unrealistic.

The rationale for Scott's model may be appreciated by considering first the far-field action of the medium, namely, the response computed on the assumption that the layer is of infinite extent and that there is no vertical wall. Each vertical strip of the medium under these conditions responds as an independent cantilever shear-beam, and no horizontal pressures develop. The resulting response, which is independent of the horizontal position coordinate, represents the particular solution of the governing equation of motion for the medium.

The response of the system with the wall may then be obtained by superimposing on the far-field or particular solution, the homogeneous solution which accounts for the effect of the normal pressures, $\sigma(\eta, t)$, exerted by the wall. The magnitude and distribution of these pressures at any time must be such that the end displacements that they induce, along with those induced by the ground shaking under free-field conditions, are equal to the wall displacements.

Let $u_{\mathrm{f}}$ be the free-field displacement relative to the moving base of a point located in the medium at a dimensionless distance $\eta=y / H$ from the base, and let $w$ be the corresponding displacement of the 
wall. These displacements are considered to be positive when directed along the positive $x$-axis. The normal wall pressures necessary to ensure compatibility of the component displacements along the wall can then be expressed symbolically by an equation of the form

$$
\sigma(\eta, t)=K(\eta, t)\left[u_{f}-w\right]
$$

where $\mathbf{K}$ is a generalized impedance or dynamic stiffness for the soil medium, defined in greater detail in the following sections. For an irrotational rigid wall, $w=0$ and the wall pressures are simply proportional to the far-field motions. Implicit in Eq. (2) is the assumption that only normal pressures develop along the wall-soil interface, that the wall can resist both compressive and tensile pressures, and that the effects of any vertical shearing stresses generated there are negligible.

Since no horizontal pressures develop under free-field conditions, the actual or total wall pressures for the base-excited system must equal those defined by Eq. (2). It follows that the expression of the wall pressure as the product of an appropriate stiffness and the difference of the wall motion and the motion of the medium at the far-field is indeed correct. The uncertainty in Scott's model lies in the choice of the medium impedance, $K$.

As a measure of the accuracy of this model, a comparison is made in Fig. 3-2 of the amplitudes of the steady-state shear at the base of the wall, $\left|Q_{b}\right|$, computed by it and two other approaches for a harmonically excited system. A uniform viscoelastic medium and a wall that is fixed against rotation at the base are considered. Poisson's ratio and the material damping factor for the medium are taken as $v=1 / 3$ and $\delta=0.1$, respectively. The shear amplitude is normalized with respect to $\rho \ddot{\mathrm{X}}_{\mathrm{g}} \mathrm{H}^{2}$, and it is plotted as a function of the frequency ratio, $\omega / \omega_{1}$, where $\omega$ is the circular frequency of the excitation and of the resulting response and $\omega_{1}$ is the fundamental natural circular frequency of the stratum when it is considered to respond as a cantilever shear-beam. For the homogeneous medium being examined, the latter frequency is given by

$$
\omega_{1}=\frac{\pi v_{8}}{2 H}
$$

in which $v_{1}=\sqrt{G / \rho}$ is the shear wave velocity for the medium. The solid line represents the nearly exact solution obtained by the approach described in later sections, whereas the short dashed line defines Scott's solution. The accuracy of the latter solution is clearly not satisfactory. Note in particular that, whereas the exact solution leads to significantly larger base shears than Scott's solution at the low and high values of the frequency parameter, the opposite is true in the intermediate range of the frequency parameter for which $\omega / \omega_{1}$ is close to unity.

\subsection{A Modification of Scott's Model}

The discrepancies in Scott's solution are due, in part, to the failure of the model to provide for the 
radiational damping capacity of the medium. If instead of a series of massless, horizontal linear springs, the medium were approximated by a series of infinitely long, linear horizontal bars with uniformly distributed mass, it is well known (see, for example, Wolf 1988) that the action of such bars for a harmonic base motion would be identical to that of a series of dashpots. On the assumption that the bars are constrained laterally and that their horizontal faces are stress-free, the equivalent damping coefficient of the dashpots, $c$, is given by

$$
c=\sqrt{\frac{2}{1-v} G \rho}
$$

The curve in long dashed lines in Fig. 3-2 represents the frequency response curve for the base shear in the wall computed on the assumption that the medium can be modeled by a series of horizontal dashpots rather than massless springs. It is observed that, while at high values of the frequency parameter, the latter modeling of the medium is definitely superior to that of the original Scott model, it too leads to unacceptable results in the lower frequency range. 


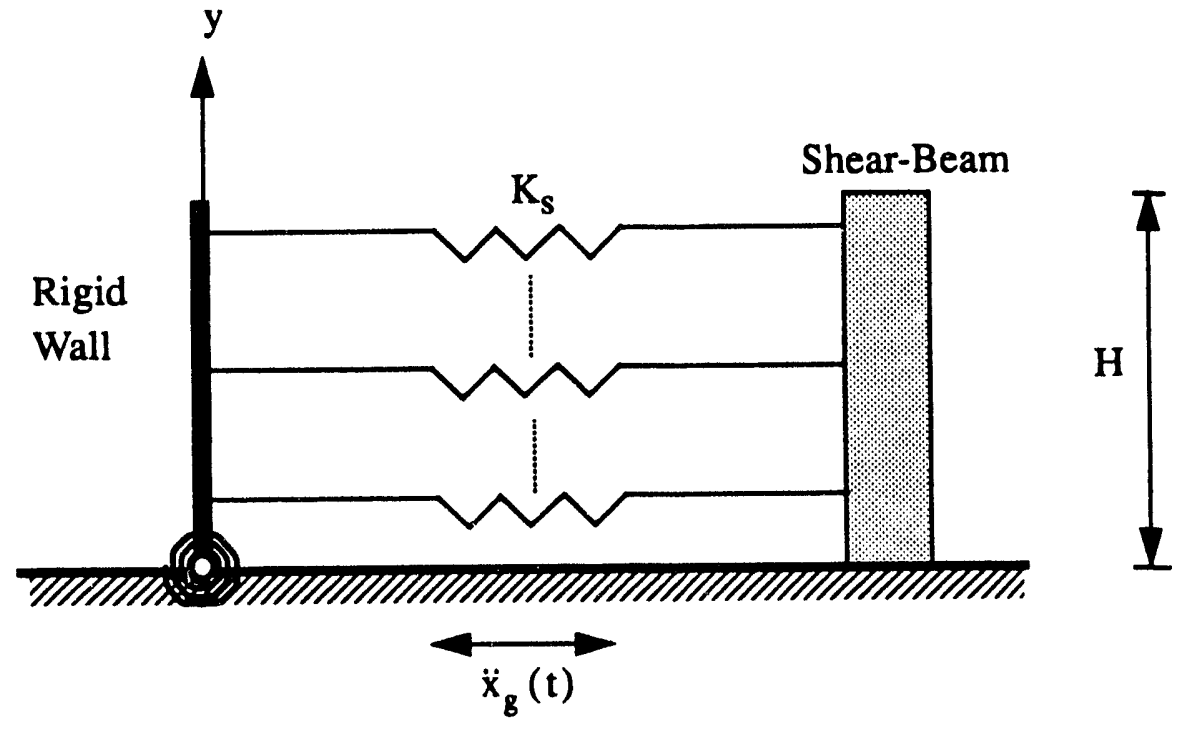

Fig. 3-1 Scott's model 


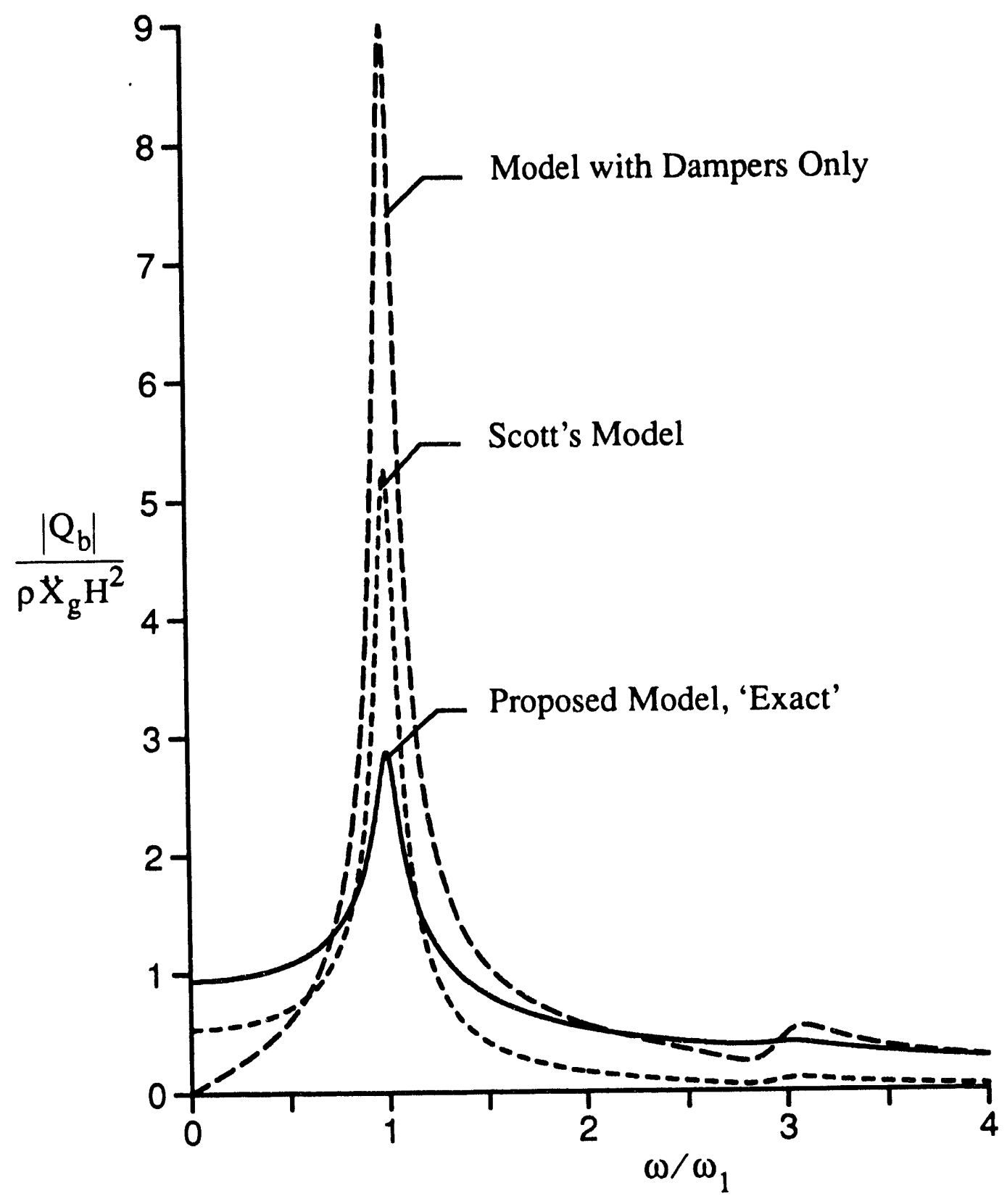

Fig. 3-2 Frequency response curves for amplitude of base shear in fixed-based wall; uniform stratum with $v=1 / 3$ and $\delta=0.1$ 


\section{SECTION 4}

\section{PROPOSED MODEL}

Both models referred to above are deficient in that they fail to provide for the capacity of the medium to transfer forces vertically by horizontal shearing action. In addition to the horizontal normal stresses and inertia forces, a horizontal element of the medium is acted upon along its upper and lower faces by horizontal shearing stresses, as shown in part (a) of Fig. 4-1, the difference of which, $\Delta \tau$, is given by

$$
\Delta \tau=\frac{\partial \tau_{x y}}{\partial y}=\frac{1}{H} \frac{\partial \tau_{x y}}{\partial \eta}
$$

On the assumption that the horizontal variation of the vertical displacements is negligible, -- a quite reasonable approximation,$-- \tau_{x y}$ can be expressed as

$$
\tau_{x y}=\frac{G}{H} \frac{\partial u}{\partial \eta}
$$

in which $u$ is the horizontal displacement of the medium relative to the moving base. On substituting Eq. (6) into Eq. (5), one obtains

$$
\Delta \tau=\frac{G}{H^{2}} \frac{\partial^{2} u}{\partial \eta^{2}}
$$

Now, let the displacement $u$ be expressed by the method of separation of variables as a linear combination of modal terms, as

$$
u(\xi, \eta, t)=\sum_{n=1}^{\infty} X_{n}(\xi) Y_{n}(\eta) q_{n}(t)
$$

in which $\xi=x / H$ is the horizontal dimensionless position coordinate, and $X_{n}, Y_{n}$ and $q_{n}$ are functions of $\xi, \eta$ and $t$, respectively. The nth component of $\Delta \tau$, denoted by $(\Delta \tau)_{n}$, may be then expressed as

$$
(\Delta \tau)_{n}=\frac{G}{H^{2}} X_{n}(\xi) Y_{n}^{\prime \prime}(\eta) q_{n}(t)
$$

which, on letting $u_{n}(\xi, \eta, t)$ represent the nth term of the right hand member of Eq. (8), can also be 
written as

$$
(\Delta \tau)_{n}=\frac{Y_{n}^{\prime \prime}(\eta)}{Y_{n}(\eta)} \frac{G}{H^{2}} u_{n}(\xi, \eta, t)
$$

For the uniform stratum under consideration,

$$
Y_{n}(\eta)=\sin \frac{(2 n-1) \pi}{2} \eta
$$

and $(\Delta \tau)_{n}$ reduces to

$$
(\Delta \tau)_{n}=-\left[\frac{(2 n-1) \pi}{2}\right]^{2} \frac{G}{H^{2}} u_{n}(\xi, \eta, t)
$$

The multipliers of $u_{n}(\xi, \eta, t)$ in Eqs. (10) and (12) are now recognized to equal $\rho \omega_{n}^{2}$, where $\omega_{n}$, the nth circular natural frequency of the stratum responding as a cantilever shear-beam, is given by

$$
\omega_{n}=\frac{n \pi v_{s}}{2 H}
$$

Accordingly, Eqs. (10) and (12) can also be written as

$$
(\Delta \tau)_{n}=-\rho \omega_{n}^{2} u_{n}(\xi, \eta, t)
$$

Equations (12) and (14) represent a force per unit of length that is proportional and opposite to $u_{n}$, the constant of proportionality being independent of the position coordinates and a function only of the order $\mathrm{n}$ of the response component being considered. Furthermore, this force is identical to that induced by a massless linear spring of stiffness

$$
k_{n}=\rho \omega_{n}^{2}=\left[\frac{(2 n-1) \pi}{2}\right]^{2} \frac{G}{H^{2}}
$$

It follows that, for each modal component of response, the horizontal shearing action of the medium may be represented by a set of horizontal linear springs of constant stiffness $k_{n}$, and that the medium may be modeled by a series of semi-infinitely long, elastically supported horizontal bars with distributed mass, as shown in part (b) of Fig. 4-1. The lower ends of all the springs are presumed to be attached to the common base experiencing the prescribed ground acceleration, $\dddot{x}_{g}(t)$. In reality, the response of only a single bar needs to be evaluated for each modal component, as the responses of all the other bars are proportional to $\sin [(2 n-1) \pi \eta / 2]$.

The steps involved in the evaluation of the response of the stratum may now be summarized as follows:

1. Compute the response of the semi-infinitely long, elastically supported bar with distributed mass considering the spring stiffness to have the value obtained from Eq. (15) with $n=1$. 
2. Determine the corresponding response of the stratum at a dimensionless distance $\eta$ from the base by multiplying the bar response by $\sin [(2 n-1) \pi \eta / 2]$.

3. Repeat steps 1 and 2 for the higher values of $n$, as necessary, keeping in mind that a change in $n$ affects both the spring stiffness $k_{n}$ and the associated natural frequency $\omega_{n}$, as well as the heightwise variation of the resulting response. It should further be recalled that the response components for the higher values of $n$ are likely to be insignificant (Veletsos and Younan 1992) and may not have to be considered.

4. Obtain the total response from Eq. (8) by superposing the component responses.

The responses obtained by this approach are identical to those obtained by the method of analysis for the stratum presented by Arias et al (1981). They are also identical to those which would have been obtained from the analysis presented by Veletsos and Younan (1992) if, in addition to assuming the absence of any vertical normal stresses, the horizontal shearing stresses had been expressed by Eq. (6) rather than by the more precise expression

$$
\tau_{x y}=\frac{G}{H}\left[\frac{\partial u}{\partial \eta}+\frac{\partial v}{\partial \xi}\right]
$$

The interrelationship of these solutions is considered further in a later section.

It is important to observe that the natural frequency of the bar in the proposed model corresponds to a mode of vibration in which the bar moves as a rigid body on springs of stiffness $k_{n}$ per unit of length. The circular value of this frequency, $\sqrt{k_{n} / \rho}$, is identical to the nth natural frequency of the stratum defined by Eq. (13). 


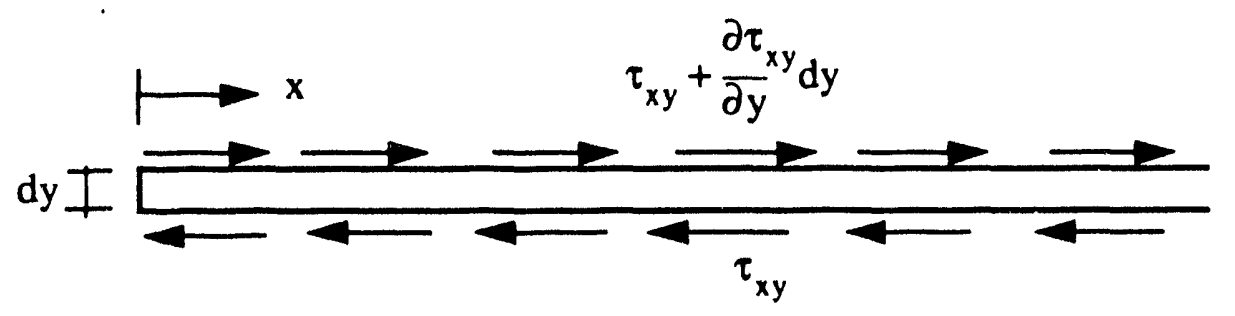

(a) State of Shearing Stresses

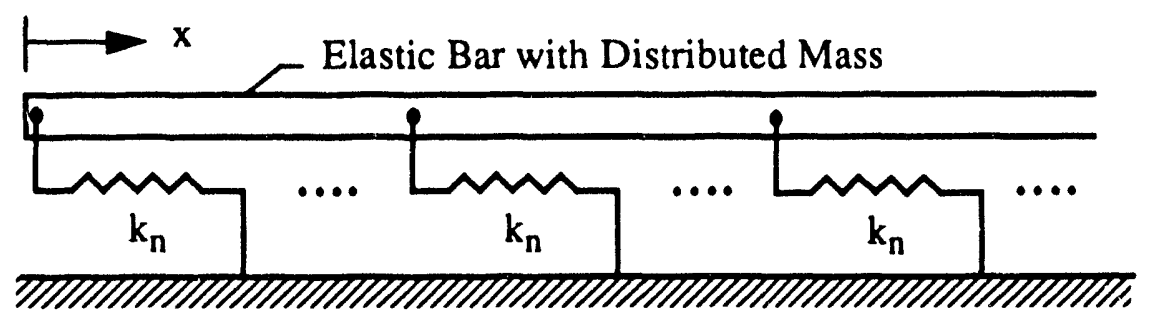

(b) Elastically Constrained Bar

Fig. 4-1 Exact modeling of soil stratum 


\section{SECTION 5}

\section{FUNDAMENTAL SOLUTIONS FOR ELASTICALLY SUPPORTED BAR}

The evaluation of the dynamic response of the semi-infinitely long, elastically supported bar with mass is a fundamental problem in foundation dynamics which has already been addressed in the literature (e.g., Wolf 1988). For an undamped elastic bar of unit cross sectional area that is stress free along its upper and lower faces and fully constrained in the lateral direction, the governing equation of motion is

$$
\tilde{E} \frac{\partial^{2} u}{\partial x^{2}}-k_{n} u=\rho \frac{\partial^{2} u}{\partial t^{2}}
$$

in which $u$ represents the displacement of an arbitrary point relative to the moving base, $\tilde{E}$ represents the laterally constrained modulus of elasticity of the bar, given by

$$
\tilde{E}=\frac{E}{1-v^{2}}=\frac{2 G}{1-v}
$$

and $E=2(1+v) G$ is Young's modulus of elasticity of the bar material. For a dissipative bar with frequency-independent, constant hysteretic damping, the real-valued shear modulus $G$ in the expressions for $k_{n}$ and $\tilde{E}$ in Eq. (17) should be replaced by the complex-valued modulus $G^{*}=G(1+i \delta)$, in which $i=\sqrt{-1}$.

\subsection{Bar Impedance}

Defined for a bar in harmonic motion, the impedance or dynamic stiffness of the bar, $K_{n}$, represents the amplitude of the harmonic end force necessary to induce a steady-state end displacement of unit amplitude. This is a complex-valued quantity that depends on the characteristics of the bar and the frequency of the excitation. For a viscoelastic bar with frequency-independent damping,

$$
K_{n}=\left(K_{s t}\right)_{n} \sqrt{(1+i \delta)\left(1-\phi_{n}^{2}+i \delta\right)}
$$

in which $\left(K_{s t}\right)_{n}$ denotes the static stiffness, defined as the static end force necessary to induce a unit end displacement, and $\phi_{n}$ denotes the dimensionless frequency ratio $\omega / \omega_{n}$. The square root sign in the latter expression and in the equations that follow refers to the first square root of tie enclosed complex-valued quantity. The static stiffness is defined by 


$$
\left(K_{n}\right)_{n}=\Psi_{0} \sqrt{G k_{n}}
$$

which, on making use of Eq. (15), can also be written as

$$
\left(K_{n t}\right)_{n}=\frac{(2 n-1) \pi}{2} \frac{\Psi_{0} G}{H}
$$

and

$$
\psi_{0}=\sqrt{\frac{2}{1-v}}
$$

Equation (19) is deduced from the corresponding equation for a laterally free, undamped bar presented by Wolf (1988) by replacing $E$ by $\tilde{E}$ and the real-valued shear modulus $G$ in the expressions for $\left(K_{n}\right)_{n}$ and $\phi_{n}$ by the complex-valued modulus $G^{*}$. This equation may more conveniently be expressed as

$$
K_{n}=\left(K_{s t}\right)_{n}\left(\alpha_{n}+i \phi_{n} \beta_{n}\right)
$$

where $\alpha_{n}$ and $\beta_{n}$ are dimensionless factors that depend on the frequency ratio, $\phi_{n}$, and the material damping factor, $\delta$. Note that the stiffness $k_{n}$ of the supporting springs in these expressions appears through Eq. (20) in $\left(K_{n 1}\right)_{n}$ and through $\omega_{n}=\sqrt{k_{n} / \rho}$ in $\phi_{n}, \alpha_{n}$ and $\beta_{n}$. It should also be noted that to each value of $n$ there corresponds a different value of $k_{n}$ and a different value of $\omega_{n}$.

The real part of Eq. (23) represents the force component that is in phase with the excitation, whereas the imaginary part represents the component that is $90^{\circ}$ out of phase. In the conventional spring-dashpot representation of the restraining action of the medium, the stiffness of the spring is represented by the real part of Eq. (23), and the coefficient of the viscous damper, $c_{n}$, is related to $\beta_{n}$ by

$$
c_{n}=\beta_{n} \frac{\left(K_{s 1}\right)_{n}}{\omega_{n}}=\beta_{n} \psi_{0} \sqrt{G p}
$$

The factors $\alpha_{n}$ and $\beta_{n}$ will be referred to as the stiffness and damping coefficients, respectively.

Fig. 5-1 shows the variations of $\alpha_{n}$ and $\beta_{n}$ as a function of the frequency ratio, $\phi_{n}$, for several different values of the material damping factor, $\delta$. Also shown for the special case of an undamped medium are the corresponding curves obtained for Scott's model for a value of $n=1$ and $v=1 / 3$, and for the model in which the action of the medium is represented by a set of viscous dampers. The lack of correlation between the results obtained by the latter two models and the exact model need not be overemphasized.

For a non-dissipative medium and values of $\phi_{n} \leq 1$ (i.e., for exciting frequencies lower than the natural frequency of the stratum for the mode of vibration being considered), the damping coefficient $\beta_{n}=0$, indicating the absence of radiation damping in this range. By contrast, for $\phi_{n} \geq 1$, the damp- 
ing coefficient is finite but the stiffness coefficient $\alpha_{n}=0$. These are well known facts that have been noted before by Wolf (1988) and by Meek and Wolf (1991) among others. 


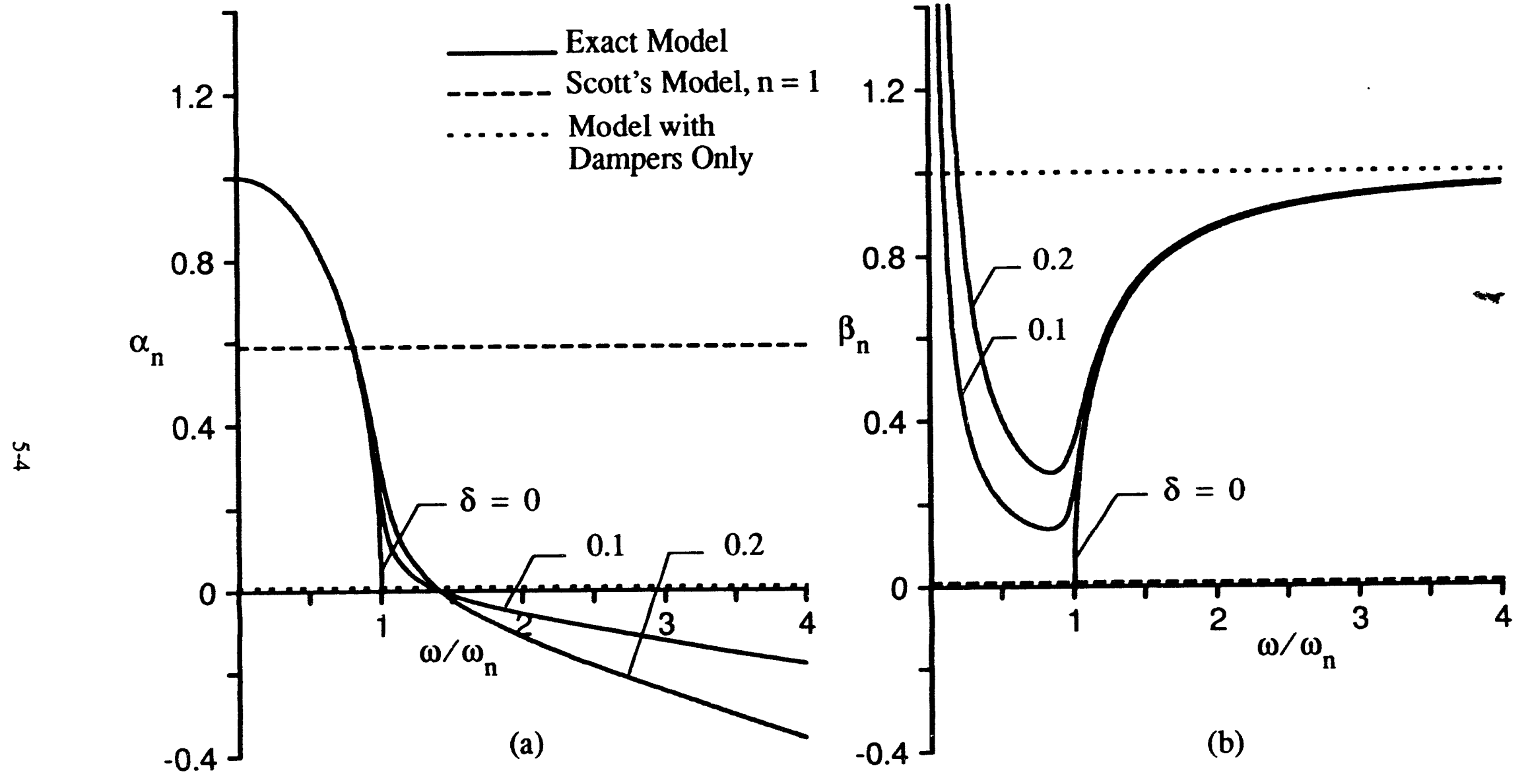

Fig. 5-1 Stiffness and damping coefficients for semi-infinite elastically constrained bar 


\section{SECTION 6 \\ HOMOGENEOUS MEDIUM WITH IRROTATIONAL WALL}

\subsection{Harmonic Response}

With the impedance of the bar established, the steady-state response of the bar to a harmonic base motion and the corresponding response of a homogeneous stratum may be evaluated readily. Let $u_{f}(\eta, t)$ be the instantaneous value of the far-field, horizontal steady-state displacement relative to the moving base of a point of the stratum located at a dimensionless distance $\eta=y / H$ from the base. This displacement may be expressed by the superposition of modal components (see, for example. Veletsos and Younan 1992) as

$$
u_{f}(\eta, t)=\sum_{n=1}^{\infty} U_{n} \sin \left[\frac{(2 n-1) \pi}{2} \eta\right] e^{i \omega t}
$$

in which

$$
U_{n}=-\frac{16}{\pi^{3}} \frac{\rho \ddot{X}_{g} H^{2}}{G} \frac{1}{(2 n-1)^{3}} \frac{1}{1-\phi_{n}^{2}+i \delta}
$$

The wall pressure at an arbitrary elevation may then be obtained by multiplying each displacement component by the corresponding bar impedance defined by Eq. (19). On so doing, one obtains

$$
\begin{aligned}
\sigma(\eta, t) & =\sum_{n=1}^{\infty} K_{n} U_{n} \sin \left[\frac{(2 n-1) \pi}{2} \eta\right] e^{i \omega t} \\
& =-\frac{8 \Psi_{n}}{\pi^{2}} \rho \ddot{X}_{8} H \sum_{n=1}^{\infty} \frac{1}{(2 n-1)^{2}} \sqrt{\frac{1+i \delta}{1-\varphi_{n}^{2}+i \delta}} \sin \left[\frac{(2 n-1) \pi}{2} \eta\right] e^{i \omega t}
\end{aligned}
$$

The shear and bending moment in the wall is finally determined by integration. In particular, the base shear per unit of wall length, $Q_{b}(t)$, is given by

$$
\begin{aligned}
Q_{b}(t) & =\int_{0}^{1} \sigma(\eta, t) H d \eta \\
& =-\frac{16 \Psi_{0}}{\pi^{3}} \rho \ddot{X}_{B} H^{2} \sum_{n=1}^{\infty} \frac{1}{(2 n-1)^{3}} \sqrt{\frac{1+i \delta}{1-\phi_{n}^{2}+i \delta}} e^{i \omega t}
\end{aligned}
$$


and the corresponding base moment, $M_{b}(t)$, is given by

$$
\begin{aligned}
M_{b}(t) & =\int_{0} \sigma(\eta, t) H^{2} \eta d \eta \\
& =-\frac{32 \psi_{0}}{\pi^{4}} \rho \ddot{X}_{8} H^{3} \sum_{n=1}^{\infty} \frac{(-1)^{n+1}}{(2 n-1)^{4}} \sqrt{\frac{1+i \delta}{1-\phi_{n}^{2}+i \delta}} e^{i \omega t}
\end{aligned}
$$

Pressures are considered to be positive when tensile; a shearing force acting on the base is positive when directed along the positive $\mathrm{x}$-axis; and the corresponding base moment is positive when clockwise.

Equations (27) through (29) may be reduced to the corresponding expressions presented by Veletsos and Younan (1992) simply by replacing the factor $\psi_{0}$ by the factor

$$
\Psi_{0}=\frac{2}{\sqrt{(1-v)(2-v)}}
$$

For $v=1 / 3$, the ratio of $\psi_{0} / \psi_{0}=0.913$. In the development of these equations no provision has been made for the inertia effects of the wall mass. For the rigid, irrotational wall considered, the additional base shear and moment due to the wall inertia are given simply by $-\mu \ddot{X}_{g} H$ and $-\mu \ddot{X}_{g} H^{2} / 2$, respectively, in which $\mu=$ the mass per unit of plan area of the wall.

The values of $\mathrm{K}_{\mathrm{n}}$ in Eqs. (27) through (29) are different for the different terms of the series. However, for systems such as the one examined in this section for which the response is clearly dominated by the fundamental mode of vibration (note the rapid convergence of the results with increasing $n$ ), excellent approximations to the base shear and base moment may be obtained by replacing $K_{n}$ by $K_{1}$ and merely multiplying the appropriate far-field response by $K_{1}$.

\subsection{Transient Response}

With the harmonic response of the elastically supported bar and of the associated stratum established, their response to an arbitrary transient excitation may be evaluated by Fourier transform (DFT) techniques. The following steps are involved in the implementation of this approach: (1) Evaluate the Fourier transform of the ground acceleration, $\ddot{x}_{g}(t) ;(2)$ compute the product of this transform and the complex-valued transfer function of the desired response; and (3) obtain the desired transient response by taking the inverse Fourier transform of the latter product. The Fourier transforms are typically computed as discrete Fourier transforms using the fast Fourier transform (FFT) algorithm and appending a sufficiently long band of zeros at the end of the forcing function so as to eliminate or reduce the aliasing errors that may be introduced (Veletsos and Ventura 1985). For the solutions presented herein, the duration of this band was taken equal to 10 times the fundamental natural period of the layer idealized as a cantilever shear-beam. 
The response to an arbitrary excitation of the elastically supported bar may also be evaluated from an integral expression analogous to Duhamel's integral for single-degree-of-freedom systems. The dynamic end pressure $\sigma_{n}(t)$ in this approach may be expressed as

$$
\sigma_{n}(t)=-\frac{8 \psi_{0}}{\pi^{2}} \rho H \frac{1}{n^{2}} A_{n}(t)
$$

where $A_{n}(t)$ is a pseudo-acceleration function which, for a non-dissipative elastic medium, is given by

$$
A_{n}(t)=\omega_{n} \int_{-\infty}^{t} \ddot{x}_{B}(\tau) J_{0}\left[\omega_{n}(t-\tau)\right] d \tau
$$

and $\mathrm{J}_{\mathrm{o}}$ is the Bessel function of the first type and zero order. This expression appears to have been first presented by Kotsubo (1959) (see also Arias et al, 1981). The wall pressure at an arbitrary elevation may then be determined from

$$
\sigma(\eta, t)=\sum_{n=1}^{\infty} \sigma_{n}(t) \sin \left[\frac{(2 n-1) \pi}{2} \eta\right]
$$

The numerical solutions obtained herein were obtained by the DFT approach.

\subsection{Numerical Solutions}

Comprehensive numerical solutions for the pressures, shears and moments induced by ground shaking in rigid walls have been presented in Veletsos and Younan (1992) for both harmonic excitations and the intense segment of an earthquake ground motion record. The reader is referred to that publication for an account of the magnitude and distribution of these effects and for an assessment of the effects and relative importance of the various parameters involved. Included in this publication also are comparisons with solutions obtained by use of the Scott model. It should be recalled that the data reported in Veletsos and Younan (1992) are $\psi_{\sigma} / \psi_{0}$ times as large as those obtained by the model presented herein and by the analysis presented by Arias et al (1981). 


\section{SECTION 7}

\section{HOMOGENEOUS MEDIUM WITH ROTATIONALLY CONSTRAINED WALL}

The rigid wall in this section is presumed to be elastically restrained against rotation at the base by a spring of stiffness $R_{\theta}$, and consideration is given to the steady-state response of the system to a harmonic excitation of the base.

The instantaneous value of wall rotation, positive when clockwise, is given by

$$
\theta(t)=\theta e^{i \omega t}
$$

in which $\Theta$ is its complex-valued amplitude, and the horizontal displacement of the wall at a dimensionless distance $\eta=y / H$ from the base is given by

$$
w(\eta, t)=\Theta \eta H e^{i \omega t}
$$

When expanded in terms of the natural modes of vibration of the stratum, $Y_{n}(\eta)$, Eq. (35) can be written as

$$
w(\eta, t)=\sum_{n=1}^{\infty} w_{n} \sin \left[\frac{(2 n-1) \pi}{2} \eta\right] e^{i \omega t}
$$

in which

$$
W_{n}=\frac{8}{\pi^{2}} \frac{(-1)^{n-1}}{(2 n-1)^{2}} \Theta H
$$

In the spirit of Eq. (2), the instantaneous wall pressure may then be expressed as

$$
\sigma(\eta, t)=\sum_{n=1}^{\infty} K_{n}\left[U_{n}-W_{n}\right] \sin \left[\frac{(2 n-1) \pi}{2} \eta\right] e^{i \omega t}
$$

in which the terms involving the displacements $U_{n}$ represent the pressure induced on a fixed-base wall and are given by Eq. (26), and the remaining terms represent the contribution of the wall rotation. The rotation amplitude $\Theta$ in Eq. (37) may be determined from the equilibrium of moments around the wall base, 


$$
M_{b}^{0}-\theta M_{b}^{1}-\frac{1}{2} \mu H^{2} \ddot{X}_{8}+\frac{1}{3} \mu H^{3} \omega^{2} \Theta=R_{\theta} \theta
$$

in which $\mathrm{M}_{\mathrm{b}}^{\mathrm{o}}$ is the moment amplitude induced by the pressures exerted on the fixed-base wall defined by Eq. (29); and $M_{b}^{1}$ is the corresponding amplitude induced by the pressures associated with a unit rotation of the wall. The latter moment is given by

$$
\begin{aligned}
M_{b}^{1} & =\frac{32 H^{3}}{\pi^{4}} \sum_{n=1}^{\infty} \frac{1}{(2 n-1)^{4}} K_{n} \\
& =\frac{16}{\pi^{3}} \Psi_{0} G H^{2} \sum_{n=1}^{\infty} \frac{1}{(2 n-1)^{3}} \sqrt{(1+i \delta)\left(1-\phi_{n}^{2}+i \delta\right)}
\end{aligned}
$$

The remaining two terms on the left-hand member of Eq. (39) represent the contribution of the wall inertia. With the roition amplitude $\Theta$ evaluated, the complex-valued amplitude of the base moment is given by the product $R_{\theta} \Theta$.

The instantaneous base shear in the wall may similarly determined from

$$
Q_{b}(t)=\left[Q_{b}^{o}-\theta Q_{b}^{1}-\mu H \ddot{X}_{g}+\frac{1}{2} \mu H^{2} \omega^{2} \theta\right] e^{i \omega t}
$$

in which $\mathrm{Q}_{\mathrm{b}}^{\circ}$, the complex-valued amplitude of the component due to the pressures acting on a fixedbase wall, is determined from Eq. (28); and $Q_{b}^{1}$, the corresponding amplitude of the component due to the pressures associated with a unit rotation of the wall, is given by

$$
\begin{aligned}
Q_{b}^{1} & =\frac{16 H^{2}}{\pi^{3}} \sum_{n=1}^{\infty} \frac{(-1)^{n-1}}{(2 n-1)^{3}} K_{n} \\
& =\frac{8}{\pi^{2}} \Psi_{0} G H \sum_{n=1}^{\infty} \frac{(-1)^{n-1}}{(2 n-1)^{2}} \sqrt{(1+i \delta)\left(1-\phi_{n}^{2}+i \delta\right)}
\end{aligned}
$$

Examination of Eqs. (39) through (42) reveals that, in addition to the parameters governing the response of the system :vith the fixed-based wall, the dynamic response of the elastically constrained wall depends on the relative mass densities of the two components, $\mu / \rho \mathrm{H}$, and the dimensionless measure of the relative flexibilities of the wall and the medium,

$$
d_{\theta}=\frac{G H^{2}}{R_{\theta}}
$$

\subsection{Numerical Solutions}

Static Effects. It is desirable to examine first the responses obtained for excitations the dominant frequencies of which are small compared to the fundamental natural frequency of the stratum (i.e., for 
motions with $\phi_{1} \rightarrow 0$ ). Such excitations and the resulting effects will be referred to as static and will be identified with the subscript st. This term should not be confused with that normally used to represent the effects of gravity forces. In the equivalent, force-excited version of the problem shown in part (b) of Fig. 2-1, the static excitation is represented by a set of horizontal body forces of constant intensity $-\rho \ddot{X}_{8}$.

Figure 7-1 shows the distributions of the wall pressures induced by a static excitation in systems with four different values of the relative flexibility factor $d_{\theta}$, including the limiting value of $d_{\theta}=0$ which corresponds to a fixed-based, irrotational wall. The wall in these solutions is presumed to be massless, and Poisson's ratio for the medium is taken as $v=1 / 3$. As might be expected, the wall pressures, and hence the wall shears and bending moments, decrease with increasing $d_{\theta}$, the reduction being substantial for the larger values of $d_{\theta}$. Increasing the flexibility of the wall reduces the horizontal axial stiffness of the medium relative to its shearing stiffness, and this reduction, in turn, increases the capacity of the medium to transfer forces vertically by horizontal shearing action. It follows that consideration of the shearing resistance of the medium is particularly important for flexible walls, and that models such as Scott's which do not provide for this resistance are specially unreliable for such systems.

The wall flexibility also modifies the distribution of the wall pressures and leads to a singularity at the top. The latter fact is consistent with the results of finite element analyses of the rocking action of a wall presented by Wood (1973). It should be clear that the contribution of the higher modes of vibration increases with increasing $d_{\theta}$, and that a rather large number of terms is required to accurately represent the pressure distribution on rotationally flexible walls.

Notwithstanding this fact, an excellent approximation to the base shear in the wall is obtained considering the contributions of the first two modes of vibration, and a reasonable approximation to the corresponding base moment is obtained using the fundamental mode of vibration only. This is demonstrated in Fig. 7-2 in which the static values of these quantities are plotted as a function of $d_{\theta}$ for a massless wall and a wall with $\mu=0.15 \rho \mathrm{H}$. For the massless wall, the solutions corresponding to the fundamental mode are shown in addition to the exact solutions. The results obtained with the first two modes are almost indistinguishable from the exact results and are not shown. It is observed that the contribution to the base shear of the higher modes of vibration (mainly second) increases in importance with increasing $d_{\theta}$. As a result, the height $h$ at which the base shear must be concentrated to yield the correct moment decreases with increasing $d_{\theta}$. For a massless wall, $h$ decreases from $0.6 \mathrm{H}$ for a fixed-based wall to $0.323 \mathrm{H}$ for a wall with $d_{\theta}=5$. The effect of the wall inertia is to increase the base shear and to decrease slightly the effective height, $h$.

Dynamic Effects. In the left part of Fig. 7-3, the real-valued amplitude of the shear at the wall base of harmonically excited systems, $\left|Q_{b}\right|$, is plotted as a function of the frequency ratio $\omega / \omega_{1}$ for three dif- 
ferent values of the relative flexibility factor $d_{\theta}$. The results are normalized with respect to $\rho \ddot{X}_{B} H^{2}$. The wall in these solutions is presumed to be massless, and Poisson's ratio and the material damping factor for the medium are taken, as before, as $1 / 3$ and 0.1 , respectively.

As would be anticipated from the static solutions presented in Fig. 7-2, the rocking flexibility of the wall base decreases the base shear. However, the decrease is by no means uniform over the full range of frequencies considered. In particular, the reduction is substantially smaller at and near resonance than under static conditions of loading. The interrelationship of the dynamic and static responses may better be appreciated from the right-hand plots of Fig. 7-3, in which the ratio of the maximum dynamic to the corresponding static shears (the amplification factor) is plotted as a function of the frequency ratio for the same three values of the relative flexibility parameter $d_{\theta}$. Of special interest are the values of the ratio at first resonance. For the fixed-base irrotational wall, it is well known that the resonant amplification factor is given by $1 / \sqrt{\delta}$ (see Arias et al, 1981, and Veletsos and Younan, 1992), which equals 3.16 for the value of $\delta=0.1$ considered. By contrast, for the rotationally flexible wall, this factor is substantially larger. This is due to the reduced capacity of the flexible wall to reflect and dissipate by radiation the waves that are transmitted to it. As $d_{\theta} \rightarrow 0$, the medium tends to respond as an unconstrained shear-beam, and the amplification factor at resonance approaches the value of $1 / \delta=10$ which is applicable to a viscously damped single-degree-of-freedom oscillator.

The same general trends also are observed in Fig. 7-4 which presents normalized values of the maximum base shear induced in the wall of a system excited by the first $6.3 \mathrm{sec}$ of the N-S component of the El Centro, Califomia earthquake of 1940. The acceleration, velocity and displacement of this record have been presented in Veletsos and Tang (1990) and are not reproduced here. The peak value of the ground acceleration is $\ddot{x}_{\mathrm{g}}=0.312 \mathrm{~g}$, and the corresponding values of the velocity and displacement are $\dot{x}_{B}=14.02 \mathrm{in} / \mathrm{sec}$ and $x_{B}=8.29$ in. The absolute maximum value of the base shear, $\left|\left(Q_{b}\right)_{\text {max }}\right|$, is normalized with respect to $\rho \ddot{x}_{8} H^{2}$ and plotted as a function of $T_{1}=2 \pi \omega_{1}$, the fundamental natural period of the layer idealized as a shear beam. As a measure of the values of $T_{1}$ that may be encountered in practice, it is noted that for shear-wave velocity values for the soil in the range between 400 and $1600 \mathrm{ft} / \mathrm{sec}$ and for values of $H$ in the range between 10 and $50 \mathrm{f}$, the value of $T_{1}$ falls in the range of $0.025 \mathrm{sec}$ to $0.5 \mathrm{sec}$. Also shown in dashed lines are the corresponding results obtained by considering the contribution of only the fundamental mode of vibration. Note that the flexibility of the wall reduces the resulting base shear, but that the reduction for a given value of $d_{\theta}$ is not nearly as large for the higher values of the plots as they are for the static values. Note further that the contribution of the higher modes of vibration (mainly second) increases in importance with increasing $d_{\theta}$. 


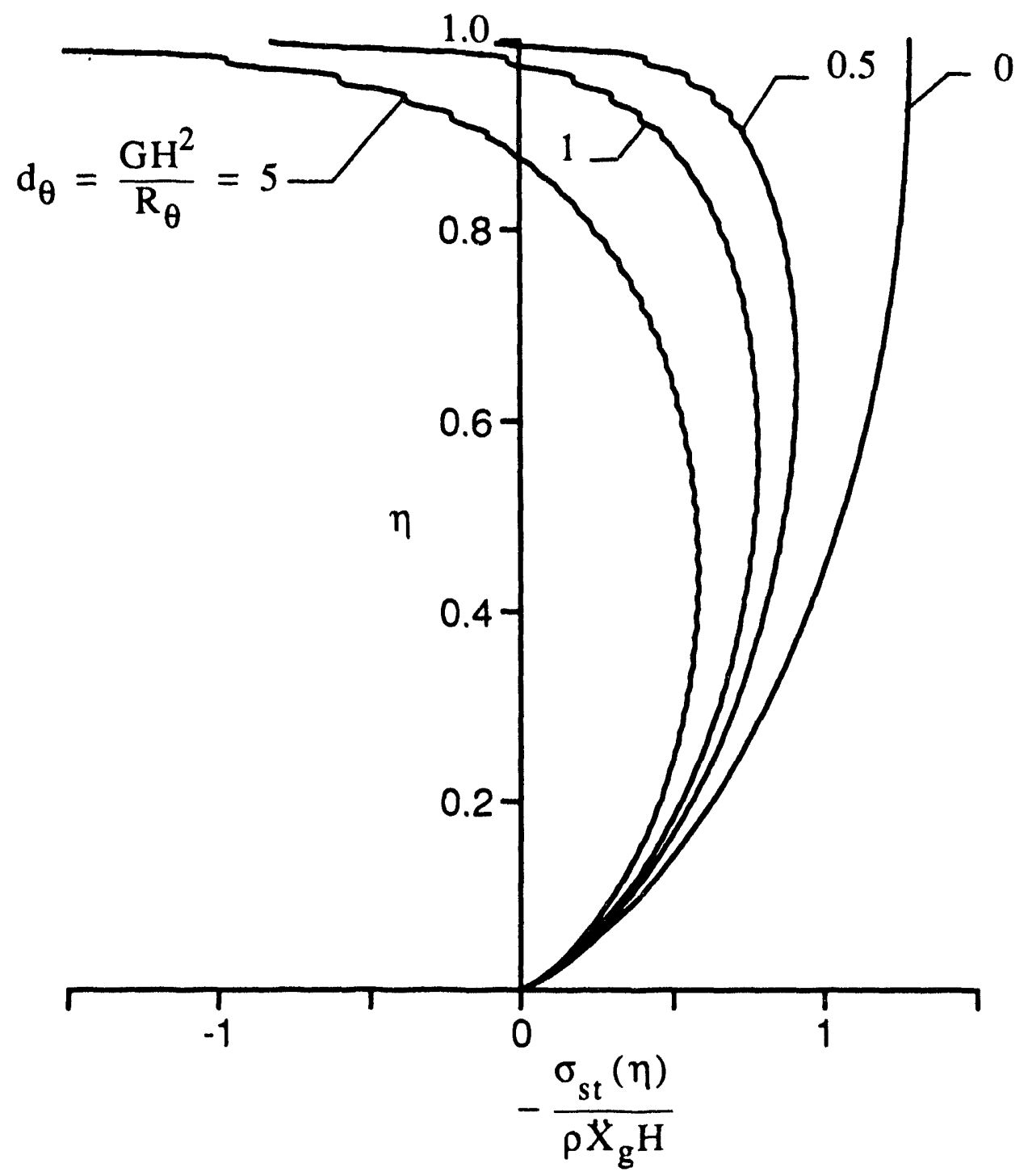

Fig. 7-1 Effect of rocking flexibility of wall on distribution of wall pressures for statically excited systems; uniform stratum with $v=1 / 3$ 


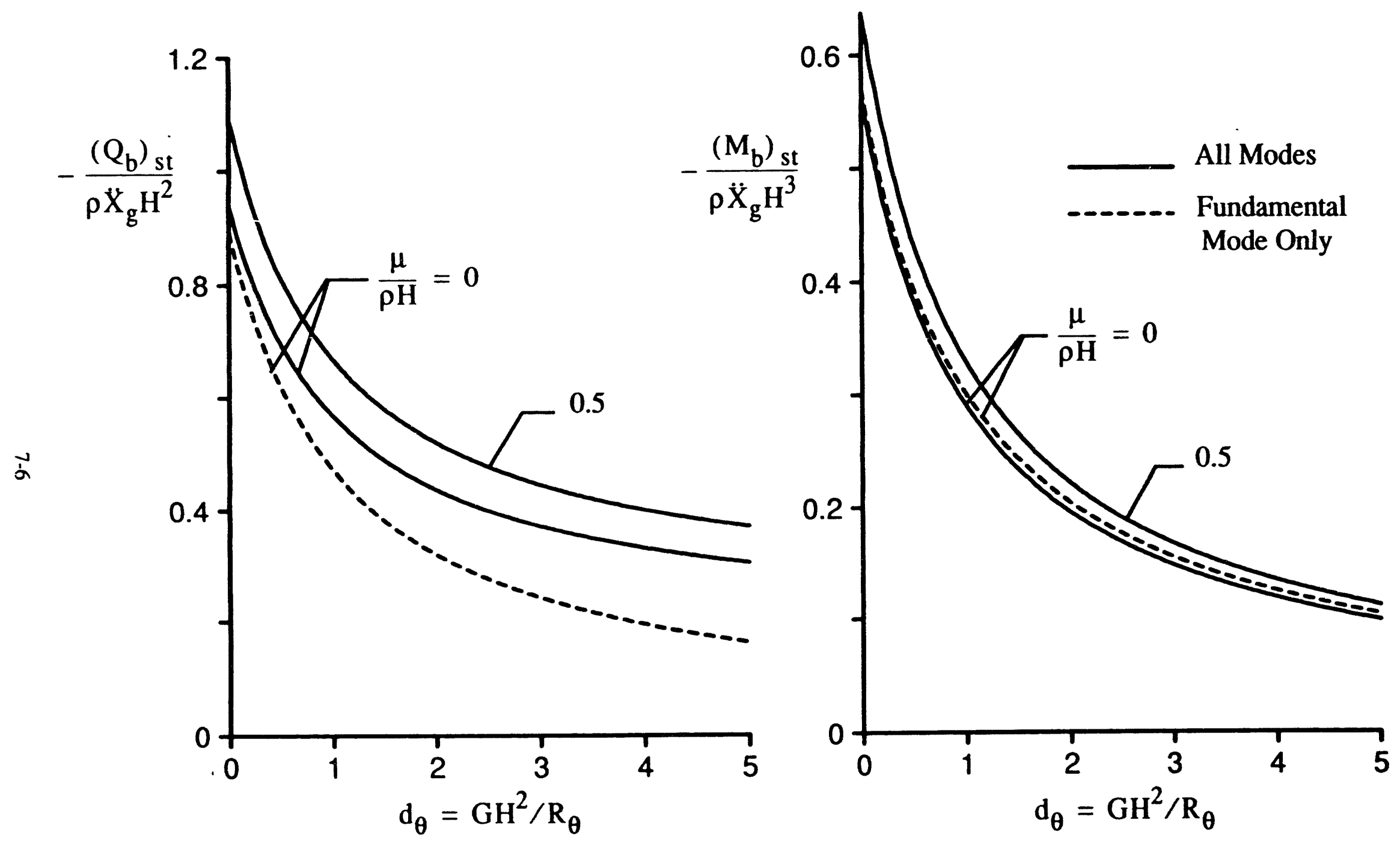

Fig. 7-2 Effect of rocking flexibility of wall on base shear and moment in wall of statically excited systems; uniform stratum with $v=1 / 3$ 


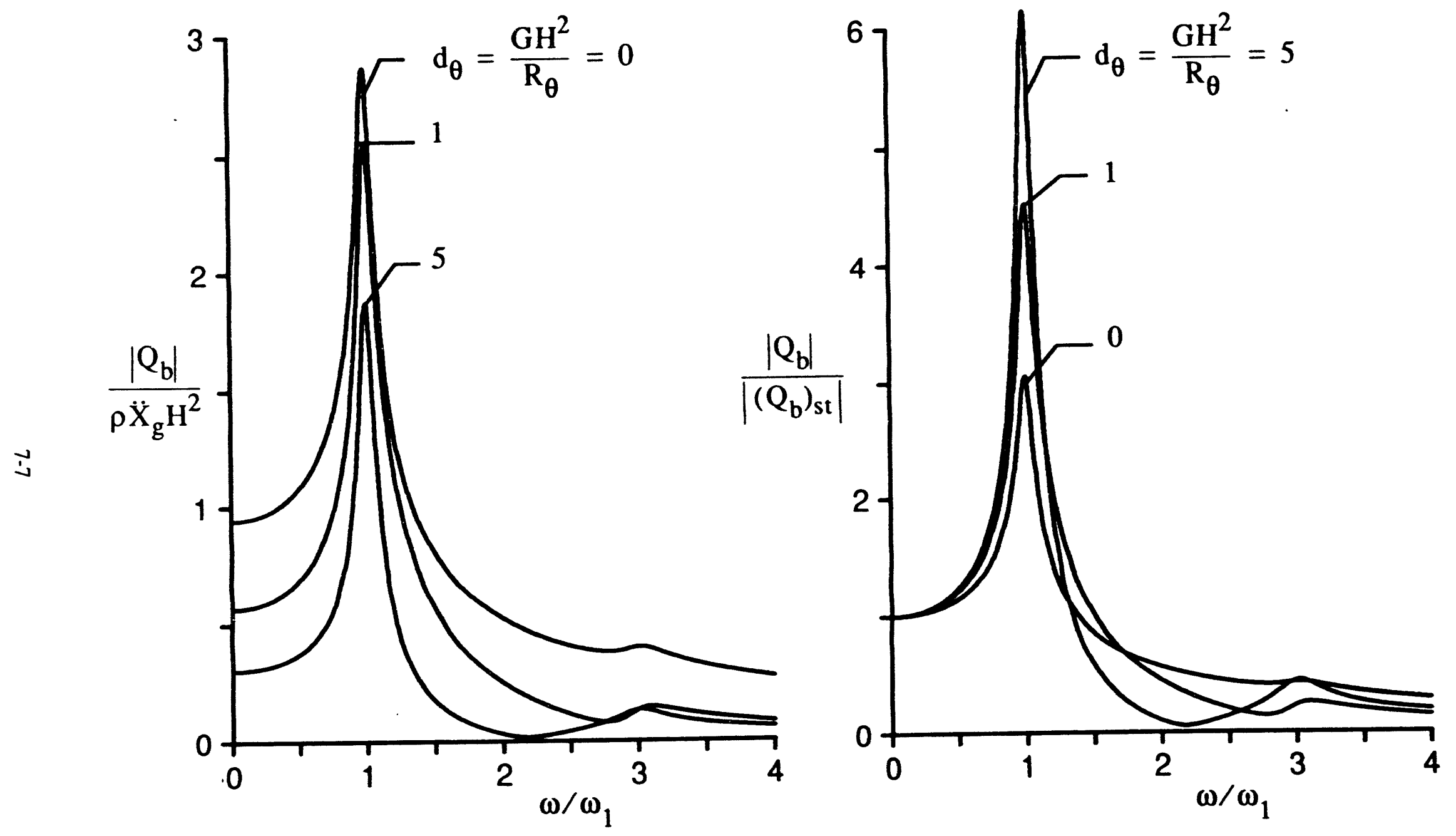

Fig. 7-3 Effect of rocking flexibility of wall on base shear of harmonically excited systems; uniform stratum with $v=1 / 3$ and $\delta=0.1$ 


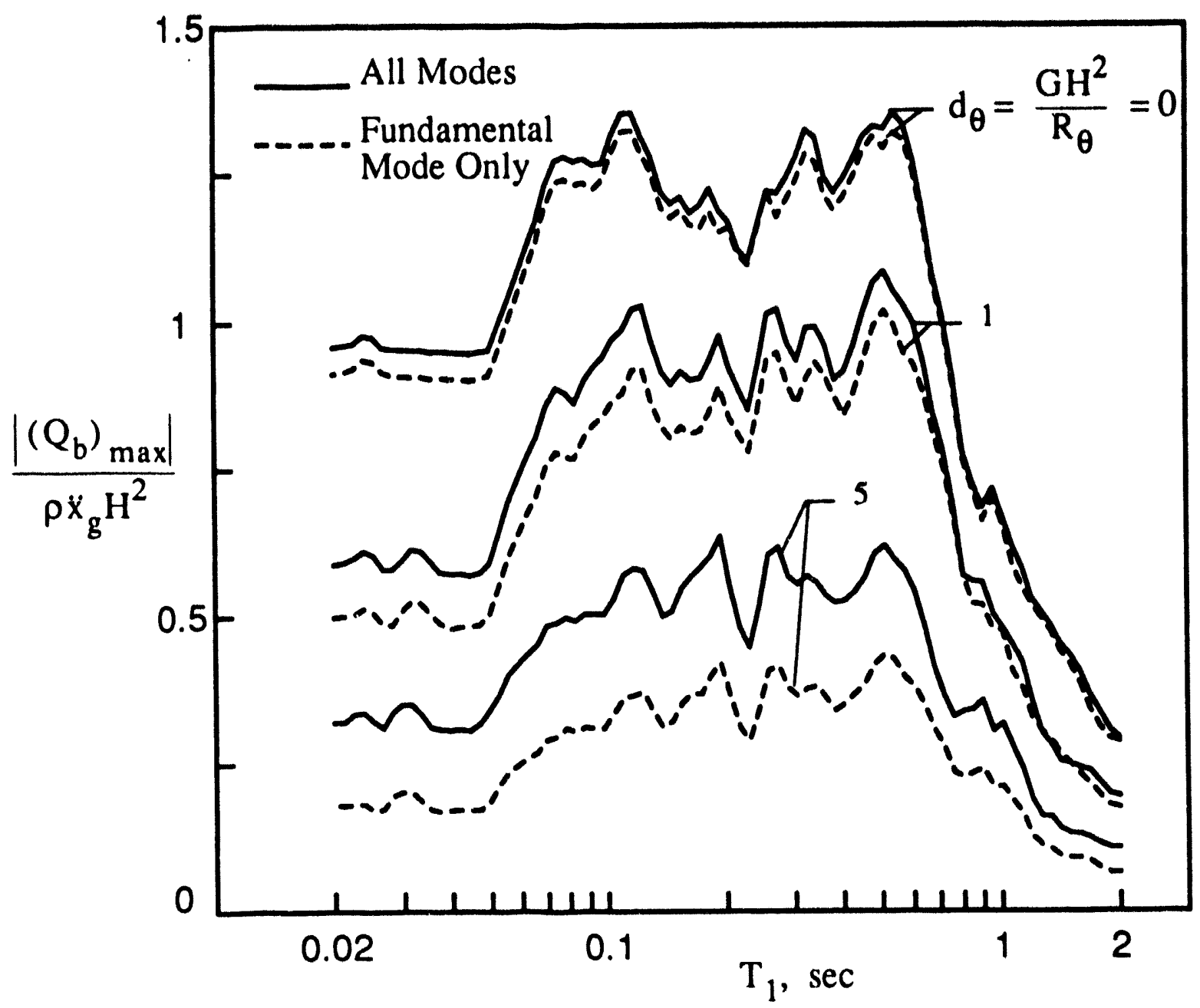

Fig. 7-4 Effect of rocking flexibility of wall on maximum base shear in wall of systems subjected to El Centro record; uniform stratum with $v=1 / 3$ and $\delta=0.1$ 


\section{SECTION 8}

\section{INHOMOGENEOUS MEDIUM WITH ROTATIONALLY CONSTRAINED WALL}

Consideration is now given to an inhomogeneous medium for which the vertical variation of the shear modulus is defined by

$$
G(\eta)=G_{0} g(\eta)
$$

in which $g(\eta)$ is a dimensionless, decreasing function of $\eta$ with $g(0)=1$, and $G_{0}$ represents the base value of the shear modulus. The mass density of the medium, $\rho$, is considered to be constant, and the wall base is considered to be elastically constrained against rotation.

The method of separation of variables employed in the solutions presented so far is not applicable in this case. As a result, the stiffnesses $k_{n}$ of the supporting springs in the modeling of the medium by elastically supported bars are in reality functions of both the $\xi$ - and $\eta$-coordinates. However, a reasonable approximation to the solution may again be obtained considering the values of $k_{n}$ to be constant and determining them from the left-hand member of Eq. (15). The frequency $\omega_{n}$ in this case must be interpreted to be the nth circular natural frequency of the inhomogeneous stratum vibrating as a cantilever shear-beam. The following considerations justify this approximation: (1) When vibrating freely in its nth natural mode, $Y_{n}(\eta)$, the difference of the shearing stresses acting on the upper and lower faces of a horizontal strip of the medium is given by Eq. (14), and Eq. (15) is exact under these conditions; and (2) for the limiting case of a statically excited medium, the solution obtained with the proposed approximation was found to be in good agreement with a more elaborate finite element solution.

As an example, consider a medium for which $G$ increases parabolically with depth as

$$
G(\eta)=G_{0}\left[1-\eta^{2}\right]
$$

The nth natural mode of vibration of this medium is given by Bielak (1969)

$$
Y_{n}(\eta)=P_{2 n-1}(\eta)
$$

in which the right-hand member represents the Legendre polynomial of order $2 n-1$, and the associated circular frequency is given by 


$$
\omega_{n}=\sqrt{2 n(2 n-1)} \frac{\left(v_{s}\right)_{0}}{H}
$$

where $\left(v_{1}\right)_{0}=\sqrt{G_{0} / p}$ is the base value of the shear wave velocity for the medium. Fig. 8-1 shows the first three modes of vibration of the medium. Notice that the top values of these modes are unity, and that the fundamental mode increases linearly from zero at the base. Accordingly, in an expansion of the displacement of the rigid wall in terms of $Y_{n}(\eta)$, only the fundamental mode will be involved.

The spring constants $k_{n}$ in this case are determined from the left-hand part of Eq. (15) to be

$$
k_{n}=2 n(2 n-1) \frac{G_{0}}{H^{2}}
$$

and, on making use of Eqs. (19) and (20), the impedance of the elastically supported bar is found to be a function of the vertical position coordinate $\eta$ and expressible as

$$
K_{n}(\eta)=\left[K_{s i}(\eta)\right]_{n}\left[\alpha_{n}+i \phi_{n} \beta_{n}\right]
$$

In the latter equation

$$
\left[K_{s t}(\eta)\right]_{n}=\sqrt{2 n(2 n-1)} \frac{\Psi_{0} G_{0}}{H} \sqrt{1-\eta^{2}}
$$

and $\alpha_{n}$ and $\beta_{n}$ are the dimensionless impedance coefficients displayed in Fig. 5-1, in which $\omega_{n}$ in the expression for $\phi_{n}$ must now be interpreted to be the nth circular natural frequency of the inhomogeneous stratum.

For a harmonically excited system, the complex-valued amplitude of the nth modal component of the steady-state displacement of the medium at the far-field, $U_{n}$, is given by

$$
U_{n}=-\frac{C_{n}}{2 n(2 n-1)} \frac{1}{1-\phi_{n}^{2}+i \delta} \frac{\rho \ddot{X}_{8} H^{2}}{G_{0}}
$$

in which

$$
C_{n}=\frac{\int_{Y_{n}}^{0}(\eta) d \eta}{\int_{0} Y_{n}^{2}(\eta) d \eta}=\frac{\sqrt{\pi}(4 n-1)}{2 \Gamma(1.5-n) \Gamma(n+1)}
$$

and $\Gamma$ is the Gamma function. The integrals in Eq. (52) were evaluated from expressions given in Abramowitz and Stegun (1965), and Gradshteyn and Ryzhik (1965). The factors $C_{n}$ represent the coefficients in an expansion of the unit function in terms of the natural modes of the stratum and, therefore, add up to unity. The first four values of $C_{n}$ are $1.5,-0.875,0.6875$ and -0.5859 , respec- 
tively.

Recalling that the wall displacement, just like the fundamental mode of vibration of the medium, increases linearly from base to top, the wall pressure at a normalized height $r_{1}$ may be written in the form

$$
\sigma(\eta, t)=\left[K_{1}(\eta)\left[U_{1}-\theta H\right] \eta+\sum_{n=2}^{\infty} K_{n}(\eta) U_{n} Y_{n}(\eta)\right] e^{i \omega t}
$$

in which $Y_{n}(\eta)$ is defined by Eq. (46), $K_{n}(\eta)$ and $U_{n}$ are determined from Eqs. (49) and (51), respectively, and $\theta$ is computed, as before, from Eq. (39). The integrals of $Y_{n}$ involved in the expressions for $M_{b}^{o}$ and $M_{b}^{1}$ in Eq. (39) and in the expressions for $Q_{b}^{o}$ and $Q_{b}^{1}$ in Eq. (41), were evaluated by numerical integration.

It is clear from Eq. (53) that, as the flexibility of the wall constraint and hence $\boldsymbol{\theta}$ increase, the first term on the right-hand member of Eq. (53) decreases, while the remaining terms, being independent of $\theta$, remain the same. It follows that the greater the flexibility of the wall constraint, the greater is the percentage contribution of the higher modes of vibration to the resulting wall pressures and the associated forces. The plots in solid lines in Fig. 8-2 show the exact distributions of the wall pressures for statically excited systems, whereas the dashed lines show those computed by considering the contribution of only the fundamental mode of vibration of the stratum. The results are for a massless wall and a medium with $v=1 / 3$. Three different values of the relative flexibility factor

$$
d_{\theta}=\frac{G_{a v} H^{2}}{R_{\theta}}
$$

are considered, in which $G_{a v}=(2 / 3) G_{0}$ is the average shear modulus of the medium. Note the following:

1. Unlike the pressure distributions for the homogeneous medium presented in Fig. 7-1 which for the rotationally flexible walls exhibit a singularity at the top, the top values of the pressures for the inhomogeneous medium are zero.

2. The pressure distributions in Fig. 8-2 bear no resemblance to the inverted triangular distribution reported for the same problem by Scott (1973).

3. As already inferred from Eq. (53), the contribution of the higher modes of vibration relative to that of the fundamental increases in importance with increasing flexibility of the wall constraint. However, reasonable approximations for the base shear and base moment in the wall may still be obtained from the first two or only the first mode of vibration.

The static values of the base shear and base moment for a medium with a parabolic variation in $G$ are compared in Fig. 8-3 with those obtained for a homogeneous medium with a value of $G=G_{a v}$. Note that the effects for the equivalent homogeneous medium are substantially larger than for the inhomo- 
geneous medium.

As a measure of the interrelationship of the dynamic responses of the two systems, the amplification factors for the steady-state base wall shear induced by a harmonic excitation are compared in Fig. 8-4 for the two extreme values of the relative flexibility factor $d_{\theta}$ considered. The normalizing frequency $\omega_{1}$ in the abscissa of these plots represents the fundamental circular natural frequency of the medium under consideration, with the result that the first resonant peak for all curves occurs at $\omega=\omega_{1}$. Note that for values of $\omega / \omega$, less than about 1.25, the amplification factors for the inhomogeneous and homogeneous media are in reasonable agreement. It follows that, even for complex earthquakeinduced ground motions for which the dominant frequencies of the oscillations in the acceleration trace of the motion are less than about 1.25 times the fundamental natural frequency of the stratum, a reasonable approximation to the maximum base shear and moment in the wall of a system with an inhomogeneous medium may be obtained from the corresponding static values by multiplying the latter values by the amplification factors applicable to a homogenous medium. The second resonant peaks in Fig. 8-4 occur at the second natural frequency of the medium being examined. For the homogencous medium, this frequency is three times the fundamental, whereas for the medium with the parabolic variation in $G$, it is $\sqrt{6}=2.45$ times as large as the fundamental. 


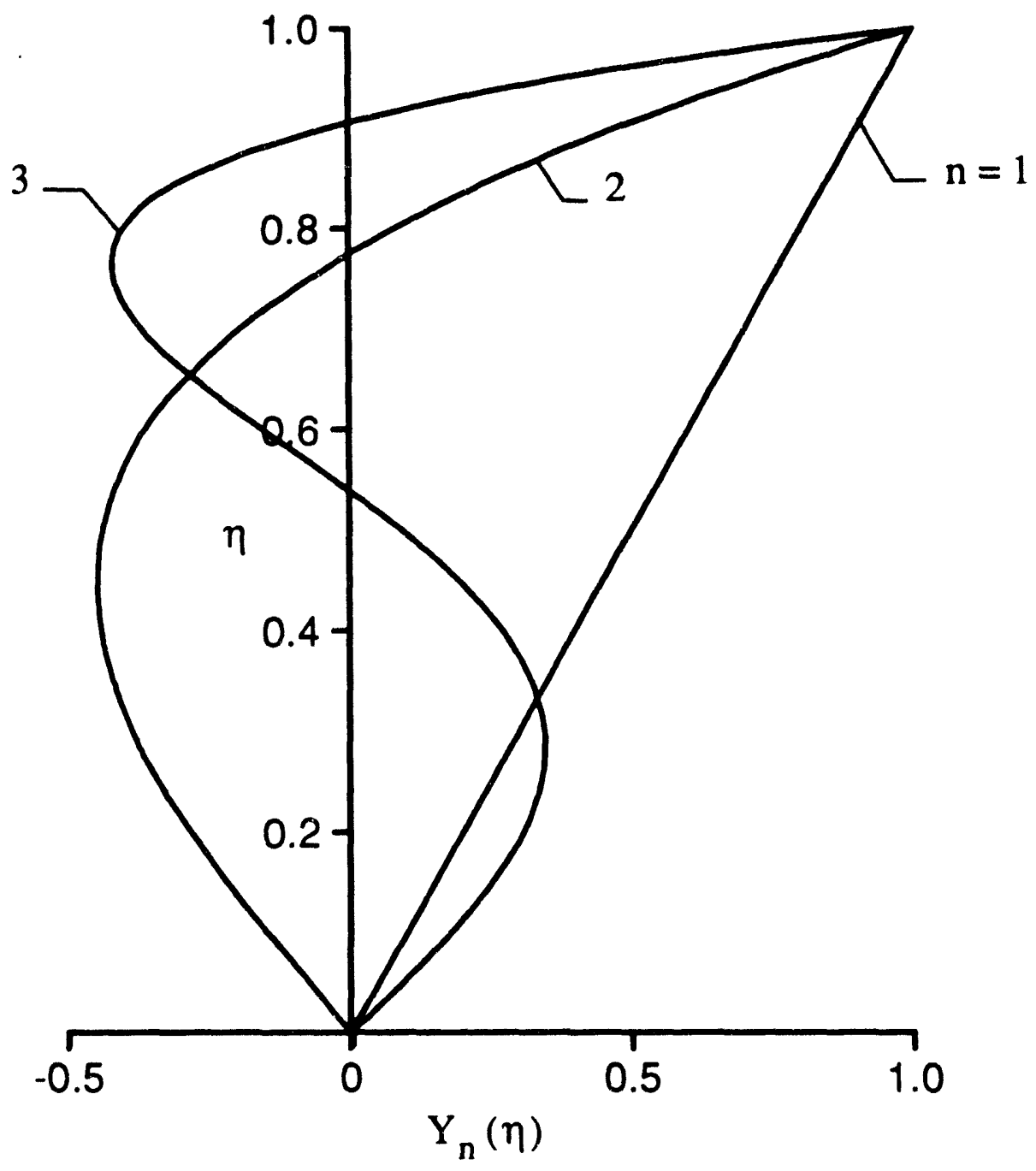

Fig. 8-1 Modes of vibration of a cantilever shear-beam with a parabolic variation of shear modulus. 


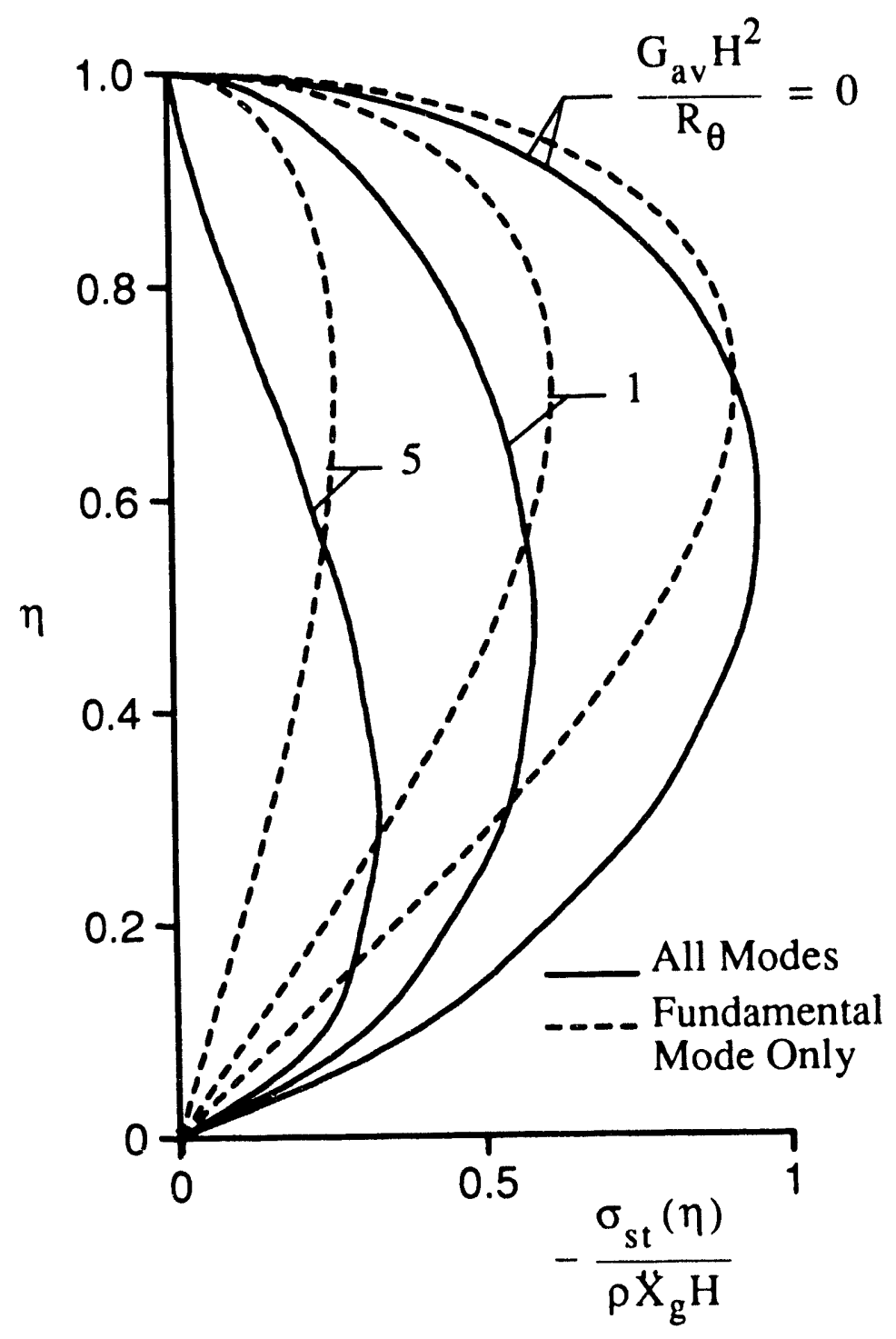

Fig. 8-2 Distribution of wall pressures for systems with a rotationally constrained wall and a medium with parabolic variation in shear modulus; $v=1 / 3$ 

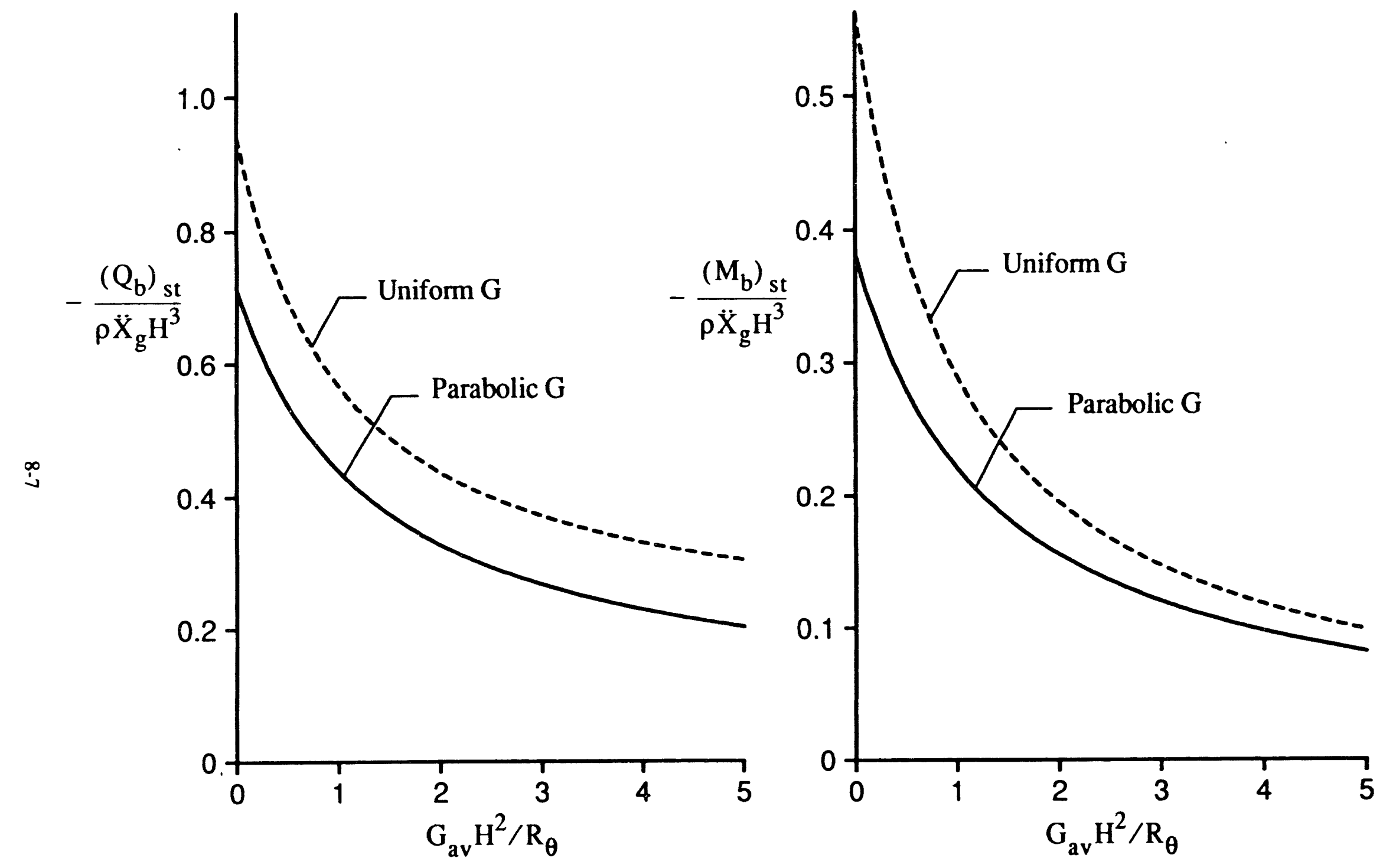

Fig. 8-3 Base shear and base moment for systems with a rotationally constrained wall and a medium with parabolic variation in shear modulus; $v=1 / 3$ 


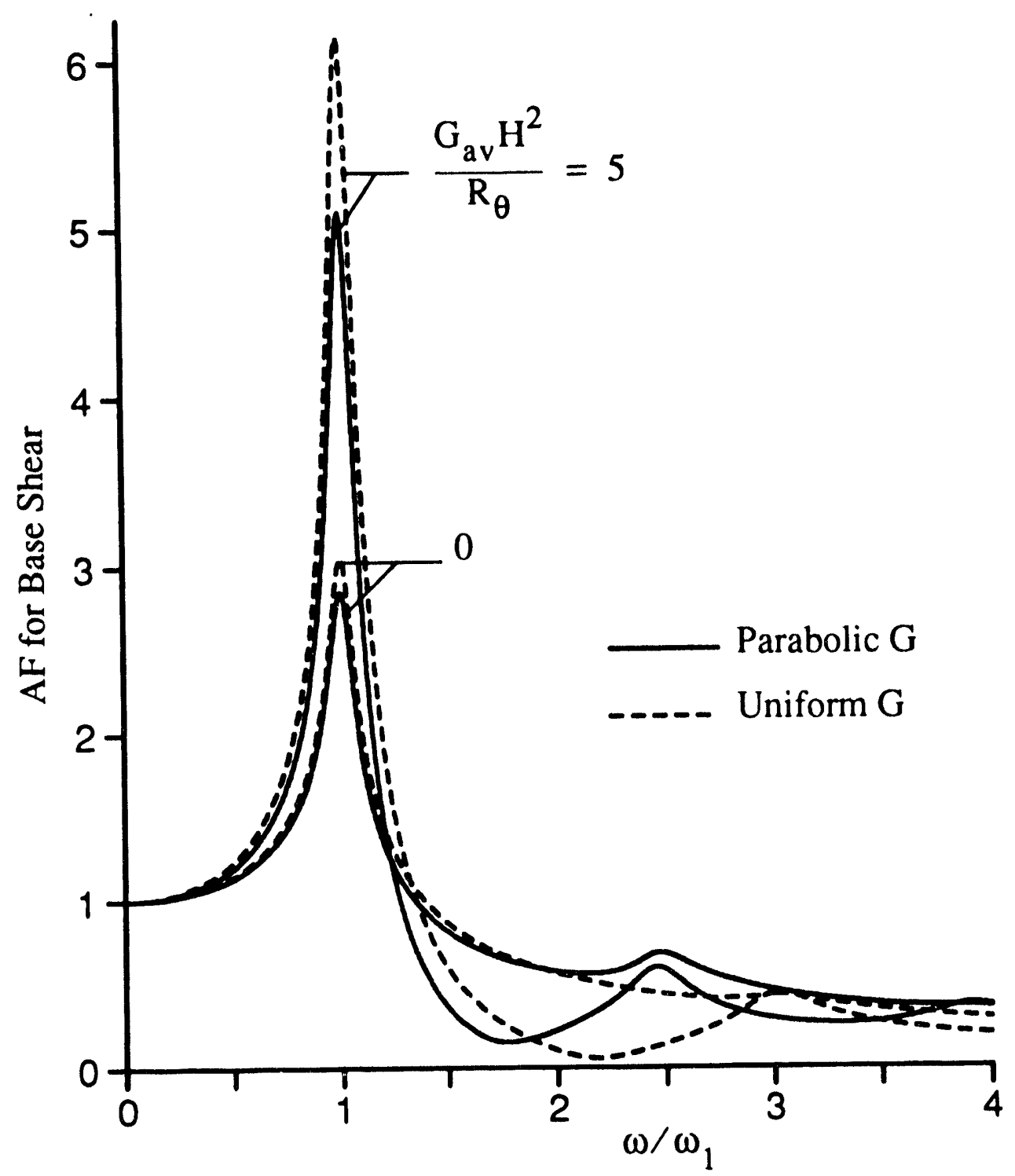

Fig. 8-4 Frequency response curves for amplitude of base shear in wall of systems with uniform, and parabolic variations in shear modulus; $v=1 / 3$ and $\delta=0.2$ 


\section{SECTION 9}

\section{CONCLUSIONS}

The principal conclusions of the study may be summarized as follows:

1. The errors in Scott's model stem from its failure to provide for the radiational damping of the medium and its capacity to transfer forces vertically by horizontal shearing action.

2. The deficiencies of this model may be elirinated by modeling the restraining action of the medium by a series of elastically supported semi-infinite horizontal bars with distributed mass rather than by massless springs. The impedance of each of these bars depends on the ratio of the exciting frequency to the natural frequency of the medium for the mode of vibration being considered, and the material damping factor of the medium. The response of the bars may be evaluated either in the frequency domain by use of Fourier transform techniques or directly in the time-domain by use of a convolution integral analogous to Duhamel's integral for single-degree-of-freedom systems.

3. The rotational flexibility of the wall decreases the dynamic wall pressures and the associated shears and bending moments and affects dramatically their distributions.

4. The greater the wall flexibility, the greater is the number of modes for the medium required to accurately represent the distribution of wall pressures. However, reasonable approximations to the base forces in the wall may still be obtained considering the contribution of only the first two modes of vibration for shear and of only the first mode for moment.

5. The magnitude and distribution of the wall pressures are also sensitive to the depthwise variation of the shear modulus for the medium.

6. The comprehensive numerical solutions presented and their analysis provide not only valuable insights into the responses of the systems examined and into the effects and relative importance of the numerous parameters involved, but also a conceptual framework for the interpretation of the responses of still more involved soil-wall systems. 


\section{SECTION 10}

\section{REFERENCES}

1. Abramowitz, M., and Stegun, I. A. (1965). Handbook of mathematical functions. Dover Publications, New York, N.Y..

2. Alampalli, S., and Elgamal, A. W. (1991). "Retaining wall; computation of seismically induced deformations." Proc. 2nd Int. Conf. on Recent Advances in Geotech. Earthquake Eng. and Soil Dyn., St. Louis, MO., I, 635-642

3. Arias, A., Sanchez-Sesma, F. J., and Ovando-Shelley, E. (1981). "A simplified elastic model for seismic analysis of earth-retaining structures with limited displacements." Proc. Int. Conf. on Recent Advances in Geotech. Earthquake Eng. and Soil Dyn., St. Louis, MO., I, 235-240.

4. Beredugo, Y. O., and Novak, M. (1972). "Coupled horizontal and rocking vibration of embedded footings." Canadian Geotech. J., 9, 477-497.

5. Bielak, J. (1969). "Base moment for a class of linear systems." J. Eng. Mech. Div. ASCE, 95, No. EM5, 1053-1062.

6. Dennehy, K. T. (1984). "Seismic vulnerability, analysis and design of anchored bulkheads." thesis presented to Rensselaer Polytechnic Institute, Troy, N.Y., in partial fulfillment of the requirement for the degree of Doctor of Philosophy.

7. Flores-Berrones, R., and Whitman, R. V. (1982). "Seismic response of end-bearing piles." J. Geotech. Eng. Div. ASCE, 108, 554-569.

8. Gradshteyn, I. S., and Ryzhik, I. M. (1965). Table of integrals, series and products. Academic Press, New York, N.Y.

9. Jain, S. K., and Scott, R. F. (1989). "Seismic analysis of cantilever retaining walls." Proc. of SMIRT, Anaheim, CA, 241-246.

10. Karkanias, S. (1983). "Seismic behavior and simplified analysis of anchored sheet pile bulkheads." thesis presented to Rensselaer Polytechnic Institute, Troy, N.Y., in partial fulfillment of the requirement for the degree of Master of Science.

11. Kotsubo, S. (1959). "Dynamic water pressure on dams due to irregular earthquakes." Memoires Fac. of Engrg., Kyushu University, 18, 4, 119-129.

12. Matuo, H., and Ohara S. (1960). "Lateral earth pressure and stability of quay walls during earthquakes." Proc. 2nd World Conf. Earthquake Engrg., Tokyo, Japan.

13. Meek, J. W., and Wolf, J. P. (1991). "Insights on cutoff frequency for foundation on soil layer." Earthquake Engrg. \& Struc. Dyn., 20, 651-665. 
14. Miller, C. A., Costantino, C. J., and Heymsfeld, E. (1991). "Soil-structure interaction effects on high level waste tanks." Proc. Third D.O.E. Natural Phenomena Hazards Mitigation Conf., St. Louis, Missouri, 588-595.

15. Novak, M., and Beredugo, Y. O. (1972). "Vertical vibration of embedded footings," J. of Soil Mech. and Found. Div. ASCE, 98, No. SM12, pp. 1291-1310.

16. Novak, M. (1974). "Dynamic stiffness and damping of piles." Canadian Geotech. J., 11, No. 4, 574-598.

17. Scott, R. F. (1973). "Earthquake-induced pressures on retaining walls." Proc. 5th World Conf. on Earthquake Eng., Rome, Italy, II, 1611-1620.

18. Soydemir, C. (1991). "Seismic design of rigid underground walls in New England." Proc. 2nd Int. Conf. on Recent Advances in Geotech. Earthquake Eng. and Soil Dyn., St. Louis, MO., I, 613-620.

19. Veletsos, A. S., and Nair, V. V. D. (1975). "Seismic interaction of structures on hysteretic foundations." J. Struct. Div. ASCE, 101, No. ST1, 109-129.

20. Veletsos, A. S., and Tang, Y. (1990). "Deterministic assessment of effects of ground-motion incoherence." J. Eng. Mech. Div. ASCE, 116, 1109-1124.

21. Veletsos, A. S., and Ventura, C. E. (1985). "Dynamic analysis of structures by the DFT method." J. Struct. Div. ASCE, 111, 2625-2642.

22. Veletsos, A. S., and Verbic, B. (1973). "Vibration of viscoelastic foundations." Earthquake Eng. \& Struct. Dyn., 2, 87-102.

23. Veletsos, A. S., and Younan, A. H. (1992). "Dynamic soil pressures on rigid vertical walls." Report No. 52357, Brookhaven National Laboratory, Upton, N.Y.; will also appear in Earthquake Eng. \& Struct. Dyn.

24. Wood, J. H. (1973). "Earthquake-induced pressures on retaining walls." Report EERL 73-05, Earthquake Engrg. Research Laboratory, Califomia Institute of Technology, Pasadena, CA.

25. Wolf, J. P. (1988). Soil-Structure Interaction Analysis in Time Domain. Prentice-Hall, Englewoods Cliffs, N.J., 88-95. 


\section{SECTION 11}

\section{NOTATION}

The following symbols are used in this study:

$A_{n} \quad$ pseudo-acceleration function defined by Eq. (32)

$\mathrm{C}_{\mathrm{n}} \quad$ nth coefficient in the expansion of a unit function in terms of the natural modes of vibration of the medium considered to respond as a series of cantilever shear beams, defined by Eq. (52)

c equivalent damping coefficient defined by Eq. (4)

E Young's modulus of elasticity of soil material

$\tilde{E} \quad$ laterally constrained modulus of elasticity of soil material, defined by Eq. (18)

G shear modulus of elasticity of soil material

$\mathrm{G}_{\mathrm{av}} \quad$ average value of shear modulus for an inhomogeneous medium

$G_{0} \quad$ base value of shear modulus for an inhomogeneous medium

$\mathrm{G}^{*} \quad$ complex-valued shear modulus for soil medium

$\mathrm{H} \quad$ height of wall and soil layer

$\mathrm{h} \quad$ height from base to resultant of total wall force

i $\quad \sqrt{-1}$

$\mathrm{J}_{\mathrm{o}} \quad$ Bessel function of the first type and zero order

$\mathrm{K}$ generalized impedance for soil medium

$\mathrm{K}_{n} \quad$ impedance of elastically constrained, semi-infinite bar with distributed mass

$\mathrm{K}_{\mathrm{s}} \quad$ stiffness of horizontal linear springs in Scott's model

$\left(\mathrm{K}_{\mathrm{st}}\right)_{\mathrm{n}} \quad$ static value of $\mathrm{K}_{\mathrm{n}}$

$k_{n} \quad$ stiffness of supporting springs for elastically constrained bar, defined by Eq. (15)

$M_{b} \quad$ instantaneous value of bending moment at wall base

$\mathrm{M}_{\mathrm{b}}^{1}$ amplitude of moment at wall base due to the pressures induced by a unit rotation of the wall

$\mathrm{M}_{\mathrm{b}}^{\mathrm{o}} \quad$ amplitude of moment at wall base due to the pressures induced on a fixed-based wall $\mathrm{n} \quad$ integer

$P_{2 n-1} \quad$ Legendre polynomial of order $2 n-1$

$Q_{b} \quad$ instantaneous value of base shear in wall

$\mathrm{Q}_{\mathrm{b}}^{1} \quad$ amplitude of base shear in wall due to the pressures induced by a unit rotation of the wall 


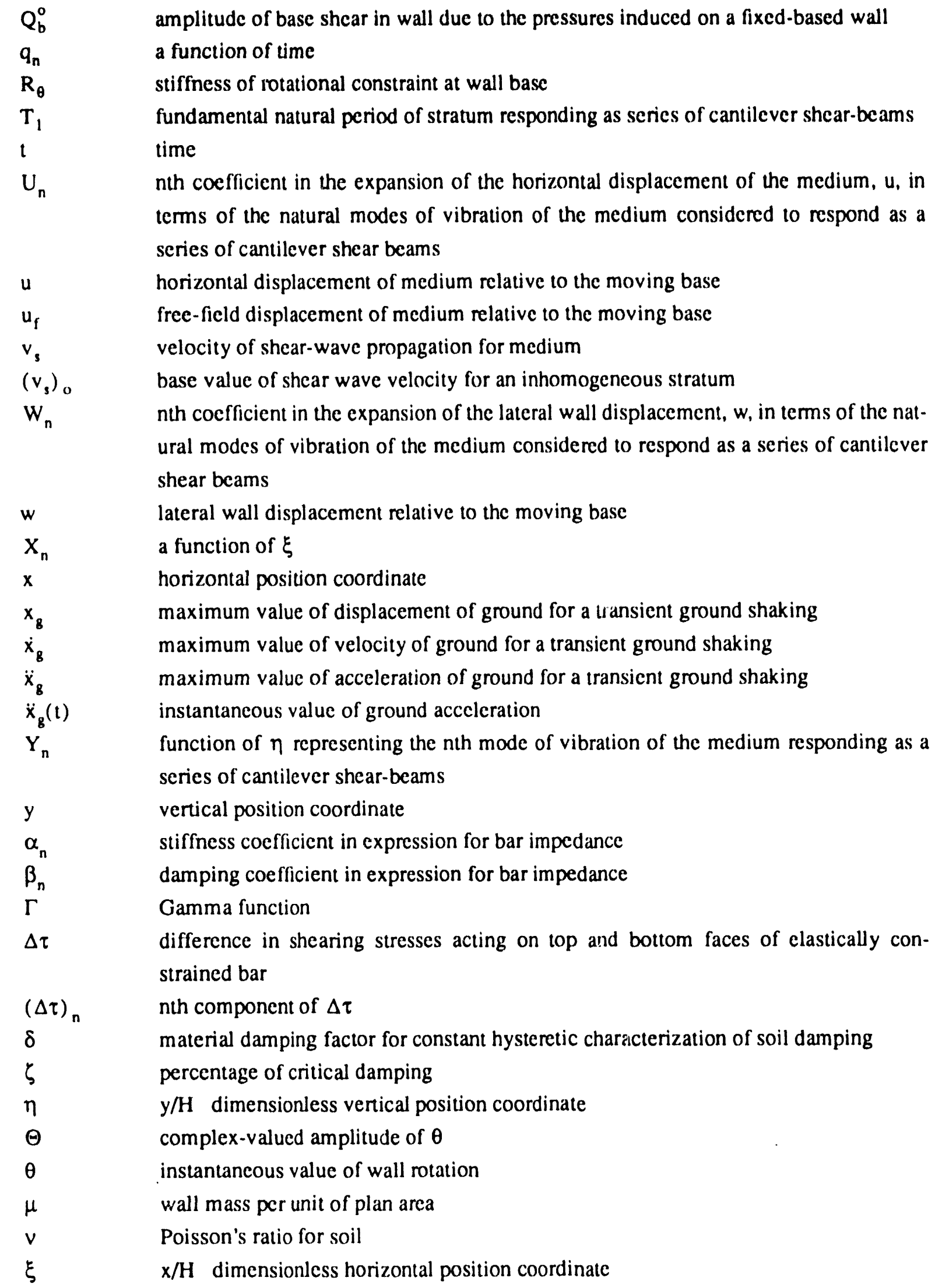


$\rho$

$\sigma$

$\sigma_{n}$

$\tau_{\text {xy }}$

$\phi_{n}$

$\psi_{0}$

$\psi_{\sigma}$

$\omega$

$\omega_{n}$

mass density for medium

instantaneous value of normal pressure induced on wall

nth component of $\sigma$

shearing stress at an arbitrary point and time

dimensionless frequency ratio

function of $v$ defined by equation (22)

function of $v$ defined by equation (30)

circular frequency of excitation and resulting steady-state motion

nth circular natural frequency of stratum considered to act as series of vertical cantilever shear-beams. 


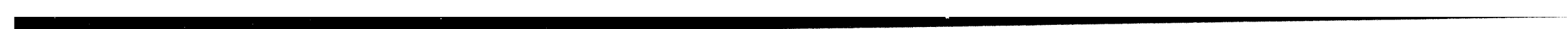




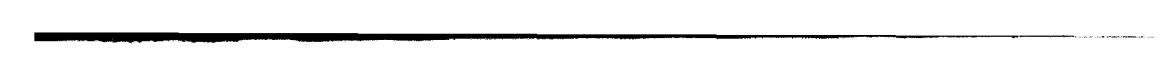

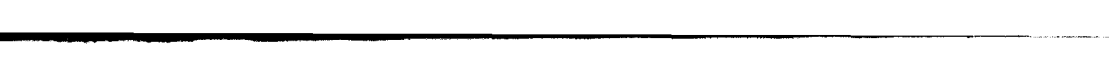
(2)

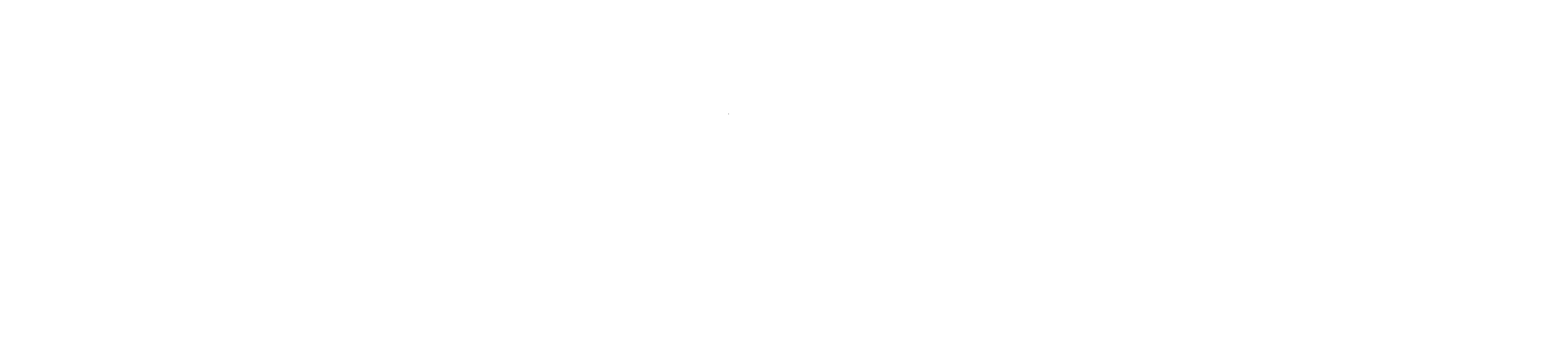

